ARTICLE

DOI: $10.1038 / \mathrm{s} 41467-017-00235-\mathrm{x}$

\title{
IL-7R $\alpha$ glutamylation and activation of transcription factor Sall3 promote group 3 ILC development
}

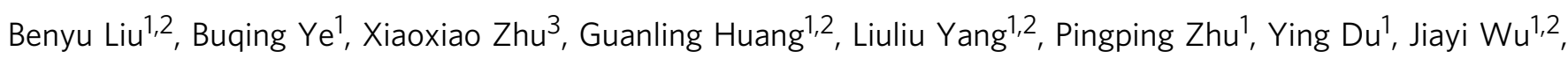
Shu Meng ${ }^{3}$, Yong Tian (1) ${ }^{2,3} \&$ Zusen Fan ${ }^{1,2}$

Group 3 innate lymphoid cells (ILC3) promote lymphoid organogenesis and potentiate immune responses against bacterial infection. However, how ILC3 cells are developed and maintained is still unclear. Here, we show that carboxypeptidase CCP2 is highly expressed in common helper-like innate lymphoid progenitors, the progenitor of innate lymphoid cells, and CCP2 deficiency increases ILC3 numbers. Interleukin-7 receptor subunit alpha (IL-7R $\alpha$ ) is identified as a substrate of CCP2 for deglutamylation, and IL-7R $\alpha$ polyglutamylation is catalyzed by polyglutamylases TTLL4 and TTLL13 in common helper-like innate lymphoid progenitors. IL-7R $\alpha$ polyglutamylation triggers STAT5 activation to initiate transcription factor Sall3 expression in common helper-like innate lymphoid progenitors, which drives ILC3 cell differentiation. Moreover, $\mathrm{Tt} / 14^{-/-}$or $\mathrm{Tt} / 113^{-/-}$mice have reduced IL-7R $\alpha$ polyglutamylation and Sall3 expression in common helper-like innate lymphoid progenitors. Importantly, mice with IL-7R $\alpha$ E446A mutation have reduced Sall3 expression and ILC3 population. Thus, polyglutamylation and deglutamylation of IL-7R $\alpha$ tightly controls the development and effector functions of ILC3s.

\footnotetext{
${ }^{1}$ Key Laboratory of Infection and Immunity of CAS, CAS Center for Excellence in Biomacromolecules, Institute of Biophysics, Chinese Academy of Sciences, Beijing 100101, China. ${ }^{2}$ University of Chinese Academy of Sciences, Beijing 100049, China. ${ }^{3}$ Key Laboratory of RNA Biology of CAS, Institute of Biophysics, Chinese Academy of Sciences, Beijing 100101, China. Benyu Liu, Buqing Ye, and Xiaoxiao Zhu contributed equally to this work. Correspondence and requests for materials should be addressed to Y.T. (email: ytian@ibp.ac.cn) or to Z.F. (email: fanz@moon.ibp.ac.cn)
} 
nnate lymphoid cells (ILCs) are part of the innate immune system $^{1,2}$. ILCs reside in the mucosal tissues and respond rapidly to pathogen infection or tissue damage via germ lineencoded receptors $^{3-5}$. ILCs can be categorized into three groups based on their signature effector cytokines analogous to the classification of $\mathrm{CD}^{+}$helper T-cell subsets ${ }^{6}$. Group 1 innate lymphoid cells (ILC1), including natural killer (NK) cells and ILC1s, function in the immune response to intracellular pathogens via secreting interferon- $\gamma(\text { IFN- } \gamma)^{7}$. Group 2 innate lymphoid cells (ILC2), including natural helper cells, nuocytes and innate helper 2 cells, enhance the resistance to helminth infection through secreting type $2 \mathrm{~T}$ helper (Th2) cell cytokines ${ }^{8}$, 9 . Group 3 innate lymphoid cells (ILC3), including lymphoid tissue inducer (LTi) cells, natural cytotoxicity receptor positive $\left(\mathrm{NCR}^{+}\right)$and $\mathrm{NCR}^{-}$ILC3s, promote lymphoid organogenesis and potentiate immune responses against bacterial infection, respectively, via producing cytokines IL-17 and IL-22 $2^{10-12}$.

All ILC cells are derived from common lymphoid progenitors (CLPs), which also differentiate to $\mathrm{T}$ and $\mathrm{B}$ cells ${ }^{13}$. ILC3s, together with other ILCs, are derived from the earliest $\alpha$-lymphoid progenitor cells ( $\alpha$ LPs, CXCR6 ${ }^{+}$integrin $\alpha_{4} \beta_{7}$-expressing CLPs) ${ }^{2}$, which differentiate into common helper-like innate lymphoid progenitor (CHILP) cells ${ }^{14}$. CHILPs generate all ILCs including LTi cells but not NK cells. Downstream of CHILP, ILC progenitors (ILCP), characterized by expression of the transcription factor (TF) PLZF, lose the ability to generate LTi cells and give rise to all ILC1, ILC2, and ILC3 subsets ${ }^{15}$. RORyt (encoded by Rorc) drives differentiation of ILC3s from their precursor ILCPs ${ }^{16}, 17$. ROR $\gamma$ t deletion causes a complete loss of ILC3s but not ILC1s or ILC2s. Of note, the cytokine receptor chain IL-7R $\alpha$ (CD127) is constitutively expressed in CHILPs and all ILCs, and forms a heterodimer with the common $\gamma$-chain of IL-2R or thymic stromal lymphopoietin (TSLP) receptor to detect IL-7 and TSLP, respectively ${ }^{14}, 18$. However, how IL-7R $\alpha$ signaling regulates the ILC development and/or maintenance still remains elusive.

Protein post-translational modifications (PTM) such as phosphorylation, glycosylation, acetylation, and ubiquitination have critical functions in the regulation of activities of target proteins by changing their chemical or structural properties ${ }^{19,20}$. Another PTM, glutamylation, adds glutamate side chains onto the $\gamma$-carboxyl groups of glutamic acid residues in the sequence of target proteins ${ }^{21-23}$. Glutamylation is catalyzed by polyglutamylases, also called tubulin tyrosine ligase-like (TTLL) enzymes 24,25 . Glutamylation is a reversible modification that can be hydrolyzed by a family of cytosolic carboxypeptidases $(\mathrm{CCPs})^{26}$. Misregulations of glutamylation contribute to several physiological abnormalities. CCP1 deficiency causes hyperglutamylation of tubulins resulting in Purkinje cell degeneration ${ }^{26,27}$. We previously demonstrated that CCP6 deficiency induces hyperglutamylation of $\mathrm{Mad}$, leading to underdevelopment of megakaryocytes and abnormal thrombocytosis ${ }^{28}$. In addition, we also show that glutamylation of the DNA sensor cGAS regulates its binding and synthase activity in antiviral immunity ${ }^{29}$, suggesting that glutamylation is involved in the regulation of immune response. However, how glutamylation regulates the development and/or maintenance of ILCs is unknown.

Here, we show that IL-7R $\alpha$ can be glutamylated by TTLL4 and TTLL13, and deglutamylated by CCP2. IL-7R $\alpha$ glutamylation enhances STAT5 activation and then promotes Sall3 transcription in CHILPs that drives the development of ILC3s. Therefore, IL-7R $\alpha$ glutamylation has a critical function in ILC3 development.

\section{Results}

CCP2 deficiency increases ILC3 numbers. We previously demonstrated that deficiency in CCP5 or CCP6 leads to susceptibility to virus infection ${ }^{29}$. CCP5 and CCP6 are required for the activation of TF IRF3 and IFN induction. We, therefore, sought to explore whether glutamylation was involved in the development of ILCs and their defense against bacterial infection. We used previously established Ccp1-6 knockout (KO) mice and further validated deletion of these genes in mouse bone marrow (BM) (Supplementary Fig. 1a). We analyzed ILC3s $\left(\mathrm{Lin}^{-} \mathrm{CD} 45^{+} \mathrm{ROR} \gamma \mathrm{t}^{+}\right)$in the small intestine lamina propria in all six deficient mouse strains and found that the number of ILC3 cells was significantly increased in $C c p 2^{-/-}$mice, but not in other CCP KO mouse strains (Fig. 1a and Supplementary Fig. 1b, c). ILC3 cells can be divided into a set of subpopulations according to their expression of CD4 and NKp46 (encoded by Ncrl) receptors, such as CD $4^{+}$ILC3s, NKp $46^{+}$ILC3s, and CD 4 ${ }^{-} \mathrm{NKp} 46^{-}$ILC3s (DN ILC3s) ${ }^{6,30}$. We then determined changes of $\mathrm{NKp} 46^{+}$ILC3s $\left(\mathrm{Lin}^{-} \mathrm{CD} 45^{+} \mathrm{ROR} \gamma \mathrm{t}^{+} \mathrm{NKp} 46^{+}\right)$and $\mathrm{NKp} 46^{-}$ILC3s $\left(\mathrm{Lin}^{-} \mathrm{CD} 45^{+} \mathrm{ROR} \gamma \mathrm{t}^{+} \mathrm{NKp} 46^{-}\right)$in $C c p 2^{-l-}$ mice. We observed that both of $\mathrm{NKp} 46^{+}$ILC3s and NKp46- ILC3s were markedly increased in $C c p 2^{-/-}$mice, but not in other CCP-deficient mouse strains (Fig. 1b and Supplementary Fig. 1d). These observations were further verified by immunofluorescence staining (Fig. 1c). By contrast, CCP2-deficient mice displayed reduced numbers of ILC1s and ILC2s (Supplementary Fig. 1e, f).

NKp $46^{+}$ILC3s substantially secrete IL-22 ${ }^{13}, 31$, which has a crucial function in the early host defense against Citrobacter (C.) rodentium infection. As expected, IL-22 secreting (IL-22 ${ }^{+}$) ILC3s were three times increased in the small intestine of $C c p 2^{-l-}$ mice compared to that of littermate wild-type (WT) mice (Fig. 1d and Supplementary Fig. 1g). However, these IL-22 ${ }^{+}$ILC3s were unchangeable in other CCP-deficient mouse strains (hereafter we used Ccp6 KO mice as a negative control) (Fig. 1d). We next infected $C c p 2^{-l-}$ or $C c p 6^{-/-}$mice with $C$. rodentium. We noticed that $C c p 2^{-t_{-}}$mice were more resistant to $C$. rodentium infection compared with their littermate WT mice (Fig. 1e-g). By contrast, $C c p 6^{-/-}$mice had comparable bacterial loads compared to their littermate WT mice. Furthermore, $C c p 2^{-/-}$mice displayed increased numbers of IL-22+ ILC3s in the small intestine after C. rodentium infection (Fig. $1 \mathrm{~h}$ ). In addition, higher expression of Il22 messenger RNA (mRNAs) in $C c p 2^{-/-}$ILC3s was further confirmed with $C$. rodentium challenge (Fig. 1i). Consistently, with IL-23 stimulation, Ccp $2^{-/-}$ILC3s produced much higher levels of IL-22 protein than WT ILC3s (Fig. 1j). By contrast, $C c p 6^{-1-}$ mice had no such effect. Taken together, CCP2 deficiency causes an increased number of ILC3s that enhance clearance of $C$. rodentium.

CCP2 deficiency potentiates ILC3 differentiation from CHILPs. We next analyzed expression patterns of CCP members in the mouse hematopoietic system. We found that Ccps displayed distinct expression profiles in different hematopoietic cell populations and their progenitors (Fig. 2a). Of note, Ccp2 was highly expressed in the CHILPs and ILC3s (Fig. 2a). Intriguingly, CCP2 deficiency led to reduced numbers of CHILPs, whereas more ILCPs in BM (Fig. 2b and Supplementary Fig. 1h), suggesting CCP2 was involved in the development of ILC3s from the stage of CHILPs. We then conducted in vitro differentiation assays. We isolated CHILPs from $C c p 2^{+/+}$and $C c p 2^{-/-}$mice and cultured them with OP9 feeder cells in the presence of murine IL-7 (25 ng/ml, Peprotech) and SCF ( $25 \mathrm{ng} / \mathrm{ml}$, Peprotech). We noticed that $C c p 2^{-/-}$CHILPs generated more ILC3s compared to $C c p 2^{+/+}$CHILPs (Fig. 2c, d and Supplementary Fig. 1i). Moreover, overexpression of CCP2 dramatically reduced the formation of ILC3s, indicating that CCP2 was implicated in the development of ILC3. $\mathrm{CoCl}_{2}$ is an agonist for CCP family proteins $^{32}$, and phenanthroline (Phen) is their pan inhibitor ${ }^{26}$. 
a

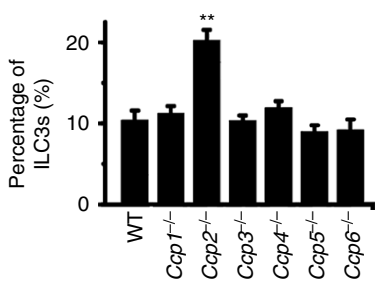

C
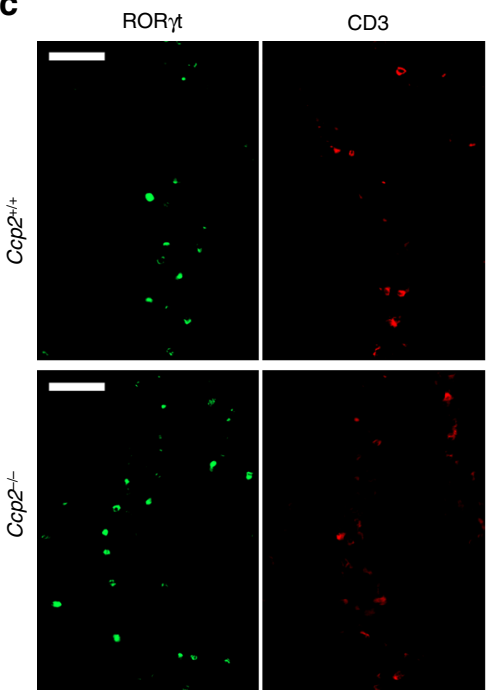

CD3

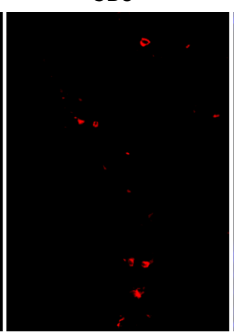

b
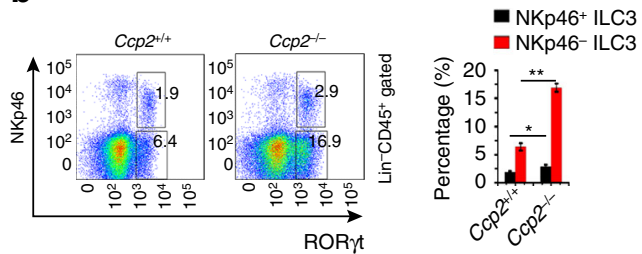

d
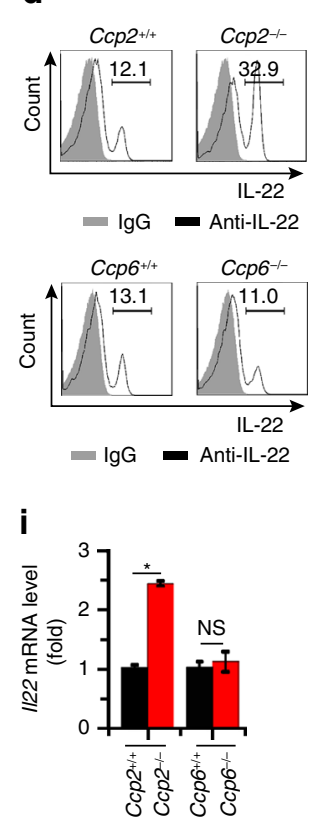
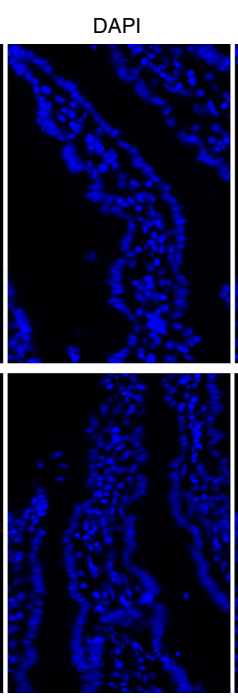

e

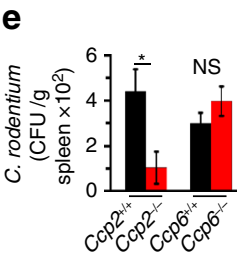

f $\mathbf{g}$

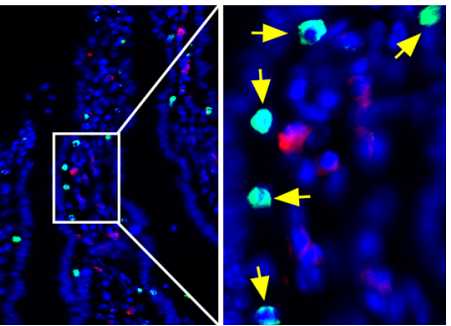

Merge
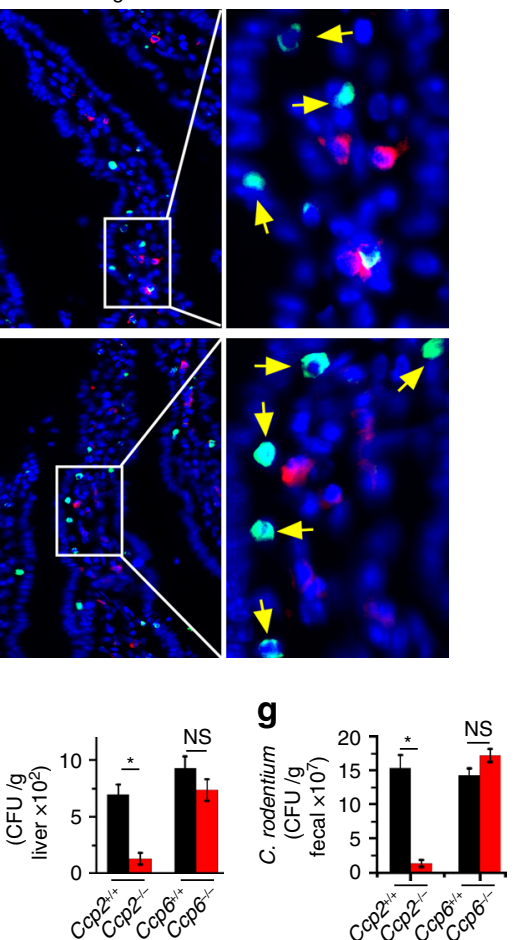

h

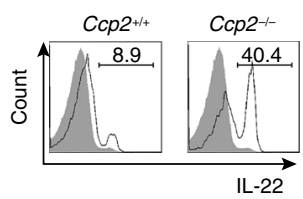

$\lg G$

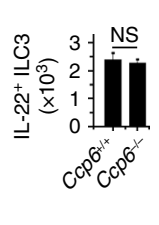

j

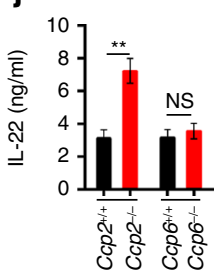

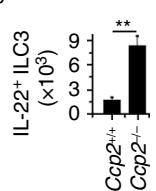
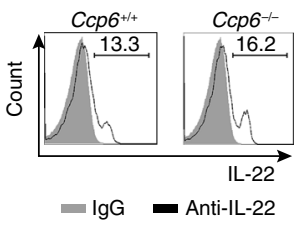

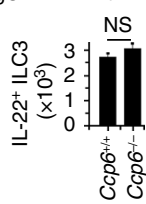

Fig. 1 CCP2 deficiency increases ILC3 numbers. a Percentages of ILC3s ( Lin $^{-}$CD45 $5^{+}$ROR $\gamma \mathrm{t}^{+}$) in small intestine lamina propria from wild-type (WT), $\mathrm{Ccp1}^{-/-}, \mathrm{Ccp} 2^{-/-}, \mathrm{Ccp3}^{-/-}, \mathrm{Ccp} 4^{-/-}, \mathrm{Ccp5}^{-/-}$, and $\mathrm{Ccp6}^{-/-}$mice were analyzed by flow cytometry. $n=6$ for each group. b Analysis of NKp46 $6^{+} \mathrm{ILC} 3 \mathrm{~s}$ $\left(\mathrm{Lin}^{-} \mathrm{CD} 45^{+} \mathrm{ROR} \gamma \mathrm{t}^{+} \mathrm{NKp} 46^{+}\right.$) and NKp46- ILC3s $\left(\mathrm{Lin}^{-} \mathrm{CD} 45^{+} \mathrm{ROR} \gamma \mathrm{t}^{+} \mathrm{NKp} 46^{+}\right.$) from $\mathrm{Ccp} 2^{+/+}$and $\mathrm{Ccp} 2^{-/-}$mice by flow cytometry. $n=6$ for each group. c Analysis of ILC3s in $\mathrm{Ccp} 2^{+/+}$and $\mathrm{Ccp2}^{-/-}$small intestines by immunofluorescence staining. Arrowhead denotes ILC3 cells. Scale bars, $50 \mu \mathrm{m}$. $\mathbf{d}$ Analysis of $\mathrm{IL}-22^{+} \mathrm{ILC} 3$ in WT, $\mathrm{Ccp} 2^{-/-}$, and $\mathrm{Ccp6}^{-/-}$small intestines after IL-23 stimulation. Cells were gated on $\mathrm{Lin}^{-} \mathrm{IL}-22^{+}$. $n=6$ for each group. e-g $\mathrm{C}$. rodentium

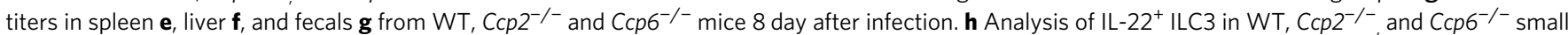
intestines after $C$. rodentium infection. $n=6$ for each group. $\mathbf{i} / 122$ expression was detected by real-time qPCR after $C$. rodentium infection. $n=6$ for each group. $\mathbf{j} 1 \times 10^{4} \mathrm{ILC} 3 \mathrm{~s}\left(\mathrm{Lin}^{-} \mathrm{CD} 45^{\mathrm{lo}} \mathrm{CD} 90^{\mathrm{hi}}\right)$ isolated from WT, $\mathrm{Ccp} 2^{-/-}$and $\mathrm{Ccp} 6^{-/-}$intestines were cultured at $37^{\circ} \mathrm{C}$ in vitro for $24 \mathrm{~h}$ in the presence of IL-23. IL-22 was examined by ELISA. $n=6$ per group. ${ }^{\star} P<0.05$, ${ }^{\star \star} P<0.01$ (Student's $t$-test). NS, no significant. Data are representative of three independent experiments. Error bars in $\mathbf{a}, \mathbf{b}$, and $\mathbf{d}-\mathbf{j}$ indicate s.d. 
a

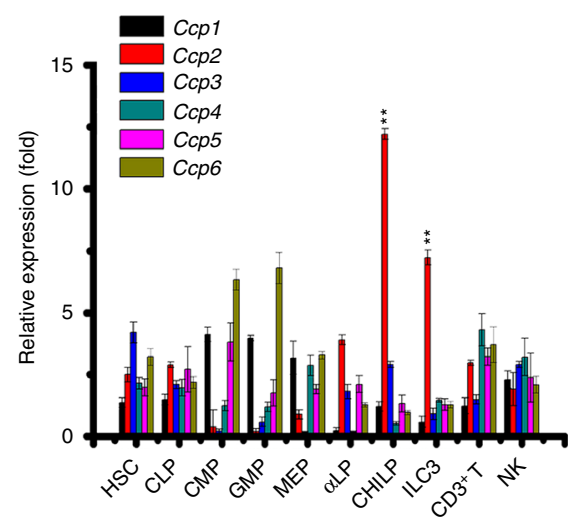

C

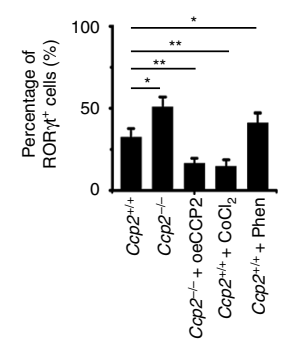

g

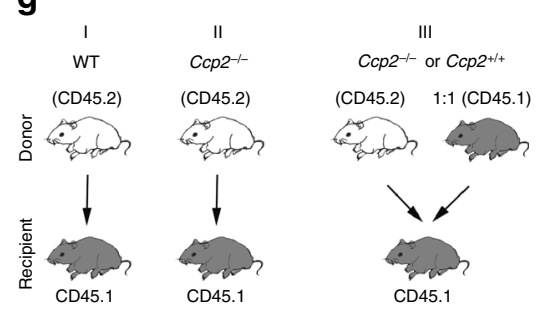

b
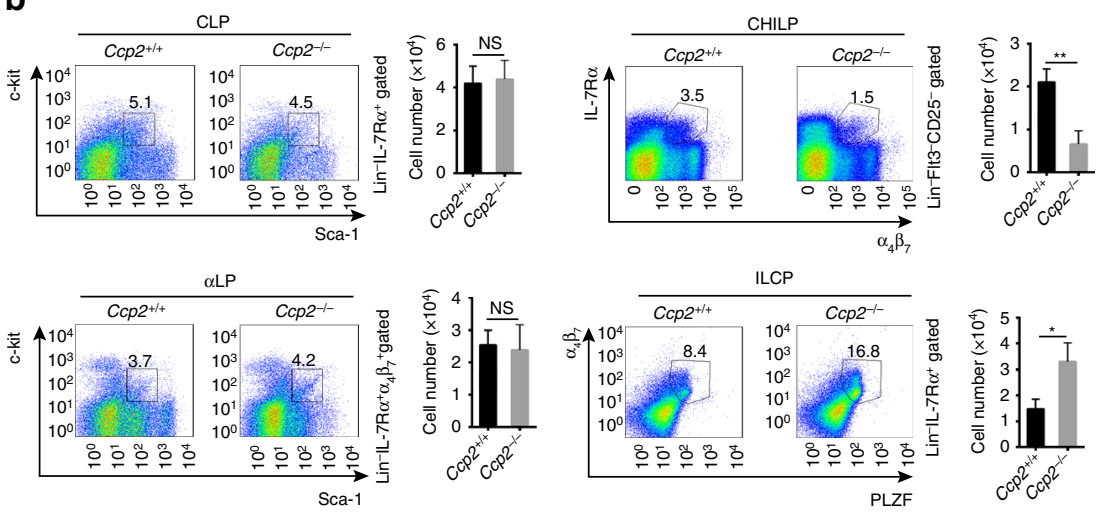

f
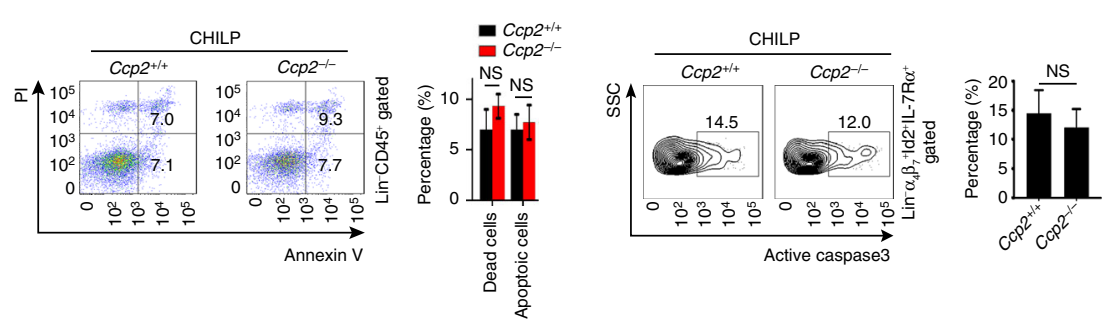

h

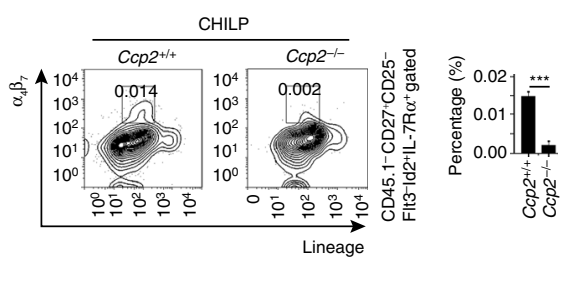

i

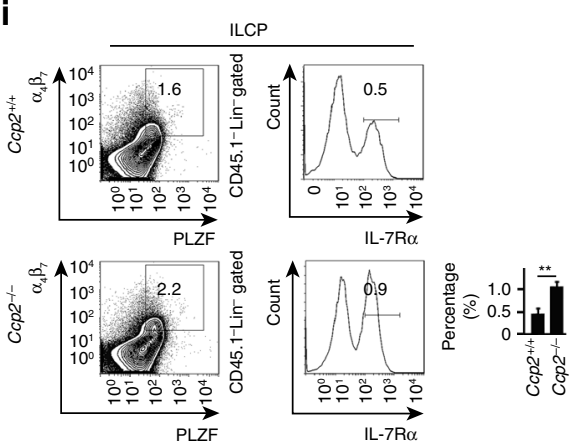

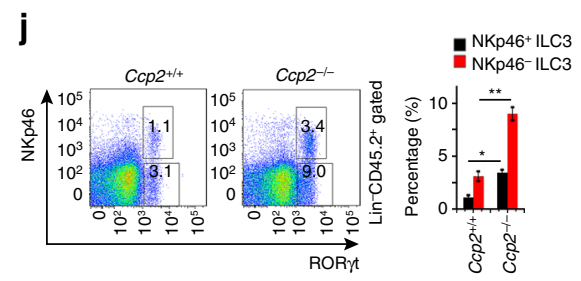

$\mathbf{k}$
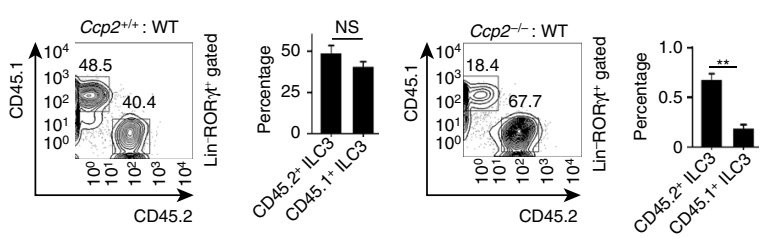

Fig. 2 CCP2 deficiency potentiates ILC3 differentiation from CHILPs. a Total RNAs were extracted from hematopoietic populations. Indicated gene expression levels of $C c p 1, C c p 2, C c p 3, C c p 4, C c p 5$, and $C c p 6$ were examined by real-time qPCR. $\mathbf{b}$ Gating strategies and flow cytometry analysis of CLP

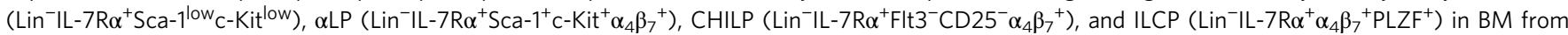

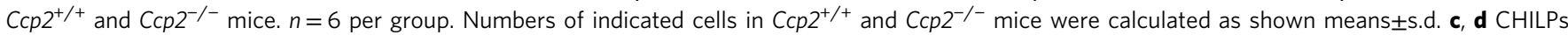
were isolated from $\mathrm{Ccp}^{+/+}$or $\mathrm{Ccp} 2^{-/-}$mice and cultured on OP9 cells in the presence of SCF and IL-7 for 12 days. Percentages $\mathbf{c}$ and total numbers $\mathbf{d}$ of ILC3s were examined by flow cytometry, gated on $\mathrm{CD}_{4} 5^{+} \mathrm{Lin}^{-} \mathrm{ROR} \gamma \mathrm{t}^{+}$. The CCP agonist $\mathrm{CoCl}_{2}(10 \mu \mathrm{m})$ and antagonist Phen $(2 \mu \mathrm{m})$ were added for in vitro differentiation assays. e Apoptosis of CHILPs from $\mathrm{Ccp}^{+/+}$and $\mathrm{Ccp}^{-/-}$mice was analyzed with Annexin V/PI. Apoptotic cells were calculated and shown as means \pm s.d. f Active caspase 3 in CHILPs from $\mathrm{Ccp} 2^{+/+}$and $\mathrm{Ccp2} 2^{-/-}$mice was detected by flow cytometry. $\mathrm{g}$ Schematic representation for BM transplantation assays. $\mathbf{h}-\mathbf{j} 5 \times 10^{4} \mathrm{CD} 45.2^{+} \mathrm{LSK}$ from $\mathrm{Ccp} 2^{+/+}$or $\mathrm{Ccp} 2^{-/-}$mice with $5 \times 10^{6} \mathrm{CD} 45.1^{+}$helper cells were transplanted into lethally irradiated CD45. $1^{+}$recipients. After 8 weeks, percentages of CHILPs $\mathbf{h}$, ILCP $\mathbf{i}$, and ILC3s $\mathbf{j}$ in chimeras were checked by FACS. $n=6$ for each group. $\mathbf{k} A 50 / 50$ mixture of $\mathrm{CD} 45.1^{+}$wild-type and $\mathrm{CD} 45.2^{+} \mathrm{Ccp} 2^{+/+}$or $\mathrm{Ccp} 2^{-/-}$bone marrow was transplanted into lethally irradiated $\mathrm{CD} 45.1^{+}$recipients. The ratio

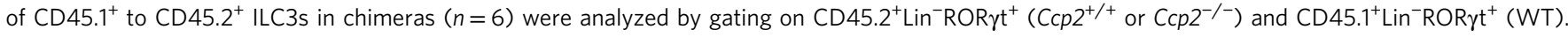
${ }^{\star} P<0.05,{ }^{\star} P<0.01$ (Student's $t$-test). Data are representative of three independent experiments. Error bars in $\mathbf{a}-\mathbf{f}$ and $\mathbf{h}-\mathbf{k}$ indicate s.d. 
a

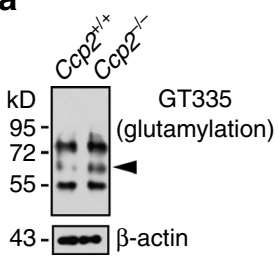

b

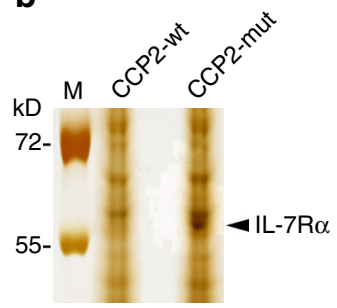

C

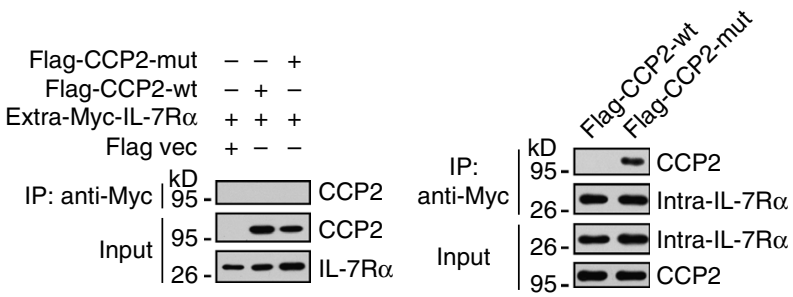

d

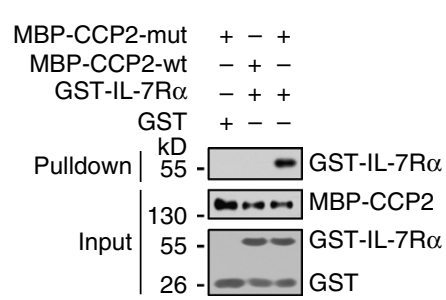

g

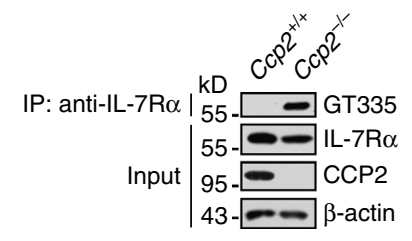

e
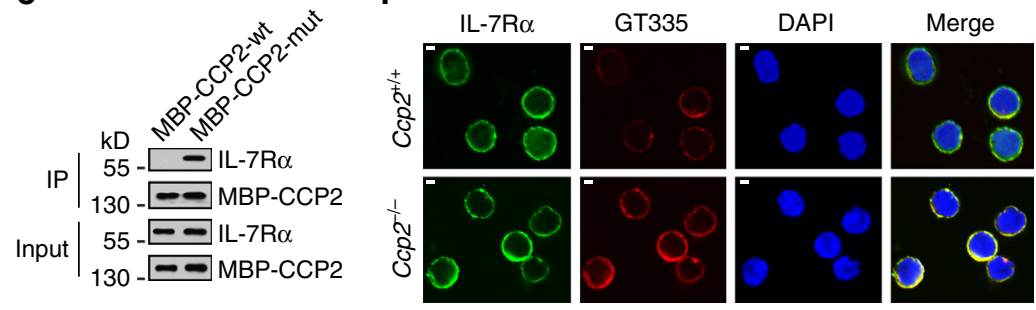

h

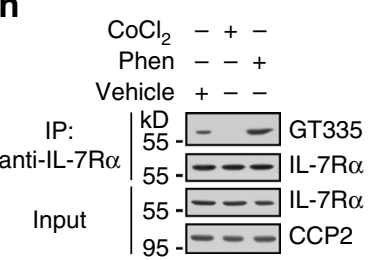

Fig. 3 IL-7R $\alpha$ is a substrate of CCP2 in CHILPs. a BM cells from WT and Ccp2 $2^{-/-}$mice were lysed. Protein glutamylation was examined with GT335 antibody by western blotting. Arrowhead denotes the differential band. b Recombinant CCP2-wt and enzymatic inactive CCP2 mutant (CCP2-mut) were immobilized with Affi-gel10 resin and assessed by addition of $\mathrm{C} c p 2^{-/-}$BM lysates. The eluted fractions were resolved by SDS-PAGE, followed by silver staining. M: molecular weight marker. A differential band of $\sim 60 \mathrm{kD}$ appeared in CCP2-mut lane and was cut for mass spectrometry. The peptide sequences and coverage of IL-7R $\alpha$ analyzed by LC-LTQ MS/MS are shown in the bottom graph. c Myc-tagged extracellular (amino acid: 21-239) or intracellular (amino acid: 265-459) segment of IL-7R $\alpha$ and Flag-tagged CCP2-wt or CCP2-mut were co-transfected in 293 T cells for $36 \mathrm{~h}$. Cell lysates were incubated with anti-Myc antibody for immunoprecipitation assay. IP immunoprecipitation. d GST-tagged intracellular segment of IL-7R $\alpha$ (GST-IL-7R $\alpha$ ) was incubated with MBP-tagged CCP2-wt or CCP2-mut at $4{ }^{\circ} \mathrm{C}$ for $4 \mathrm{~h}$, followed by incubation with GST beads. e CCP2-wt and CCP2-mut were incubated with BM lysates for pulldown assay. $\mathbf{f}$ CHILPs were incubated with GT335 and anti-IL-7R $\alpha$ antibodies for immunofluorescence staining. IL-7R $\alpha$, green; GT335, red; nucleus, blue. Scale bar, $2 \mu \mathrm{m}$. $\mathbf{g}$ BM lysates from WT or $\mathrm{Ccp} 2^{-/-}$mice were immunoprecipitated with anti-IL-7R $\alpha$ antibody, followed by immunoblotting. h WT BM cells were treated with $\mathrm{CoCl}_{2}$ or Phen. Cells were lysed and IL-7R $\alpha$ glutamylation was assessed. Data represent four independent experiments

As expected, $\mathrm{CoCl}_{2}$ treatment suppressed the generation of ILC3s, whereas Phen treatment increased the formation of ILC3s (Fig. 2c, d). Finally, Ccp $2^{-/-}$CHILPs did not undergo apparent apoptosis (Fig. 2e, f). Altogether, polyglutamylation is required for the differentiation of ILC3s from their progenitor CHILPs.

Cell-intrinsic modulations of ILC3 differentiation by CCP2. We next sought to determine whether CCP2 deficiency-mediated ILC3 development was intrinsic or extrinsic. We transplanted CD45.2 $2^{+} C c p 2^{-/-}$or $C c p 2^{+/+}$BM cells into lethally irradiated CD $45.1^{+}$recipients (Fig. $2 \mathrm{~g}$ ). Eight weeks after transplantation, $C c p 2^{-/-}$BM transferred recipients displayed reduced number of CHILPs but increased counts of ILCPs compared with those of $C c p 2^{+/+}$BM transplantation (Fig. 2h, i). ILC3s were consequently increased in $C c p 2^{-/-}$BM transferred recipients (Fig. 2j). We also performed competitive $\mathrm{BM}$ transplantation assays. We transferred a 1:1 mixture of CD45.1 $1^{+}$WT and CD 45.2 $2^{+} C c p 2^{+/+}$or $C c p 2^{-/-}$BM into lethally irradiated recipient mice (Fig. $2 \mathrm{~g}$ ). Eight weeks after transplantation, reconstituted recipients showed increased numbers of ILC3s (Fig. 2k). Collectively, CCP2 is an intrinsic factor in the regulation of ILC3 development.

IL-7R $\alpha$ is a substrate of CCP2 in CHILPs. To further explore the molecular mechanism of CCP2-mediated ILC3 differentiation, we analyzed lysates of $C c p 2^{+/+}$and $C c p 2^{-/-}$BM by immunoblotting with a glutamylation-specific antibody GT335. The antibody GT335 specifically recognizes the branch points of glutamate side chains and detects all glutamylation forms of target proteins ${ }^{26}$. After immunoblot analysis, one band around $60 \mathrm{kD}$ appeared in the lane of CCP2-deficient BM lysates (Fig. 3a). This band was undetectable in the corresponding lane location from the littermate control BM lysates. Thus, this band could be a potential candidate substrate for CCP2. To identify the candidate substrates of CCP2, we generated an enzymatically inactive mutant of CCP2 (CCP2-mut) through H425S and E428Q mutations as previously described ${ }^{26}$. WT CCP2 (CCP2-wt) and CCP2-mut were immobilized with Affi-gel10 resin to go through mouse BM lysates for affinity chromatography. The eluted fractions were resolved by sodium dodecyl sulfate polyacrylamide gel electrophoresis (SDS-PAGE), followed by silver staining. This band was present in the gel analyzing CCP2-mut and was cut for mass spectrometry, whose band was identified as IL-7R $\alpha$ (Fig. 3b and Supplementary Fig. 2a).

We transfected Myc-tagged intracellular (amino acid: 265-459) or Myc-tagged extracellular segment (amino acid: 21-239) of IL-7R $\alpha$ with Flag-tagged CCP2-wt or CCP2-mut into 293 T cells for co-immunoprecipitation assay. We found that the Flag-tagged enzymatic dead CCP2 (Flag-CCP2-mut) could pull down the Myc-tagged intracellular segment of IL-7R $\alpha$ (hereafter we called 
IL-7R $\alpha$ ) (Fig. 3c). By contrast, Flag-CCP2-mut failed to precipitate Myc-tagged extracellular segment of IL-7R $\alpha$ (Fig. 3c). Consistently, glutamylated GST-tagged intracellular segment of IL-7R $\alpha$ protein could pull down MBP-CCP2-mut by a pulldown assay (Fig. 3d), suggesting the intracellular segment of IL-7R $\alpha$ was deglutamylated by CCP2. Moreover, MBP-tagged mutant CCP2 (MBP-CCP2-mut) was able to pull down native IL-7R $\alpha$ from BM lysates, whereas the enzymatic active CCP2 (MBP-CCP2-wt) could not precipitate IL-7R $\alpha$ (Fig. 3e). These observations suggest that the intracellular segment of IL-7R $\alpha$ undergoes deglutamylation by CCP2. With immunofluorescence staining, hyperglutamylation of IL-7R $\alpha$ appeared in primary CCP2-deficient CHILPs (Fig. 3f). In parallel, IL-7R $\alpha$ was highly polyglutamylated in BM lysates of $C c p 2^{-l-}$ mice (Fig. $3 g$ ). Finally, BM cells treated with the CCP antagonist Phen increased substantial amounts of polyglutamylated IL-7R $\alpha$, whereas treatment with the CCP a

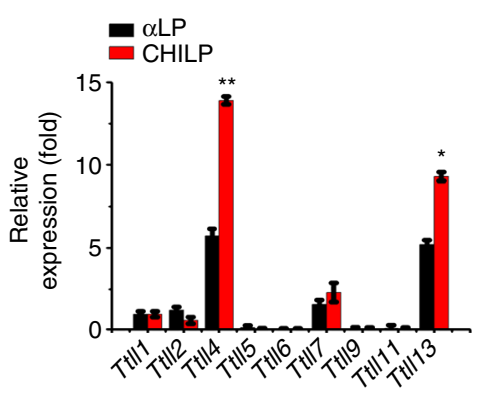

b

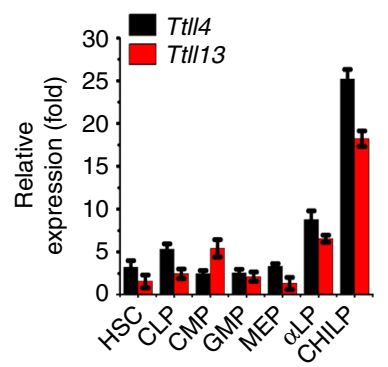

C

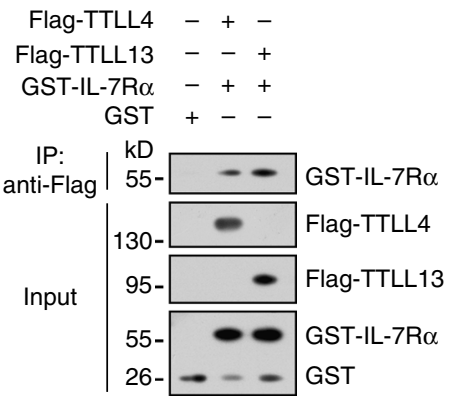

f
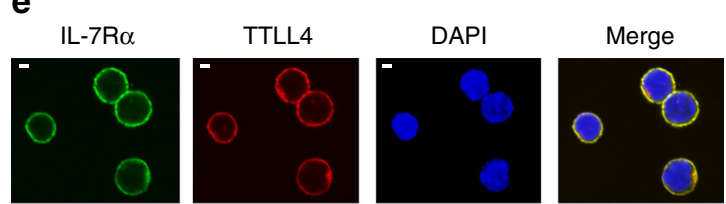

GST-IL-7R $\alpha+++$

Flag-TTLL4 - + -

Flag-TTLL13 - - +

Flag vec + - -

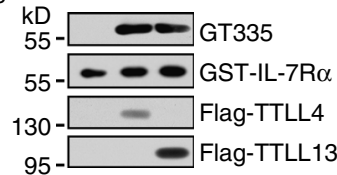

IL-7R $\alpha$

TTLL13
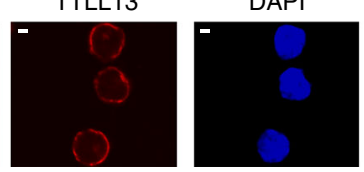

Merge

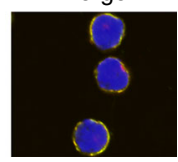

i
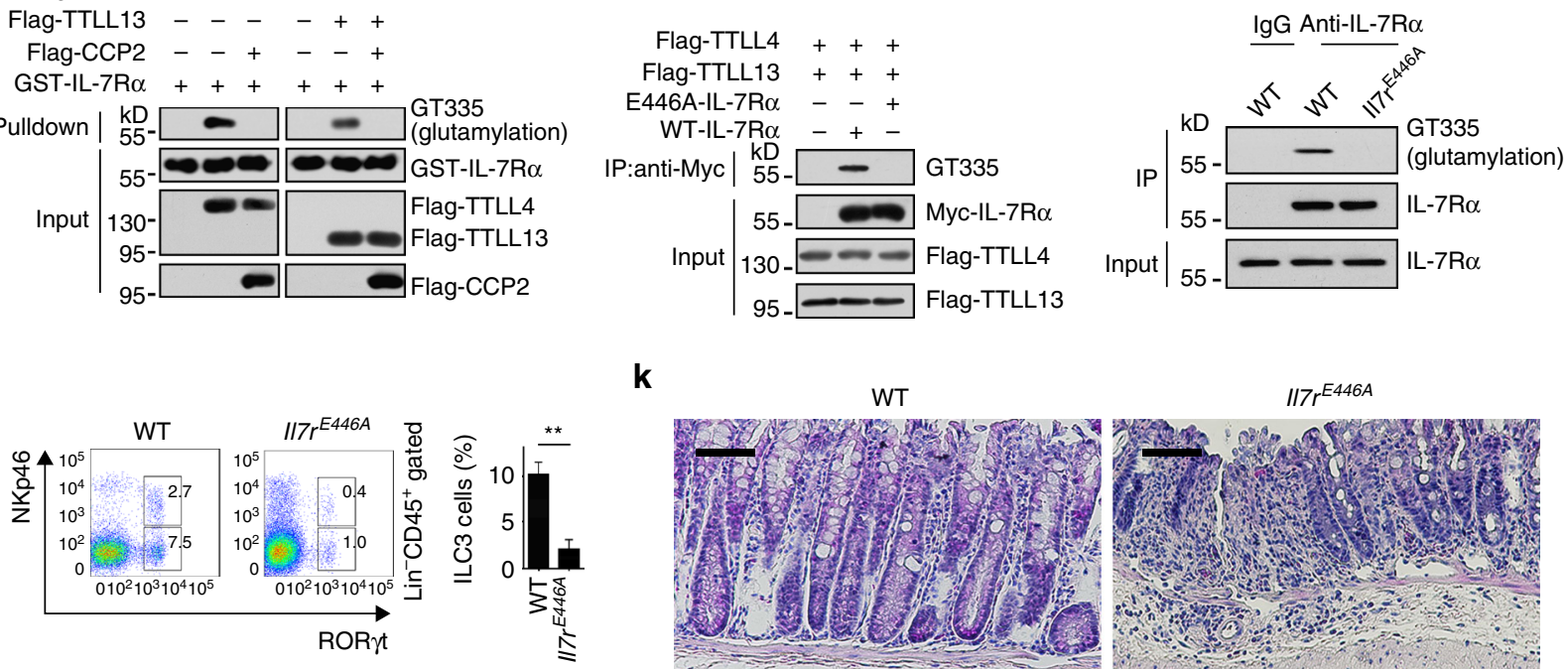

I

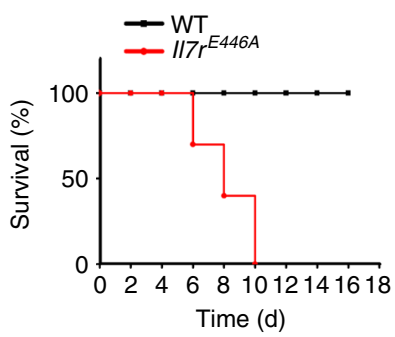

$\mathbf{k}$

h
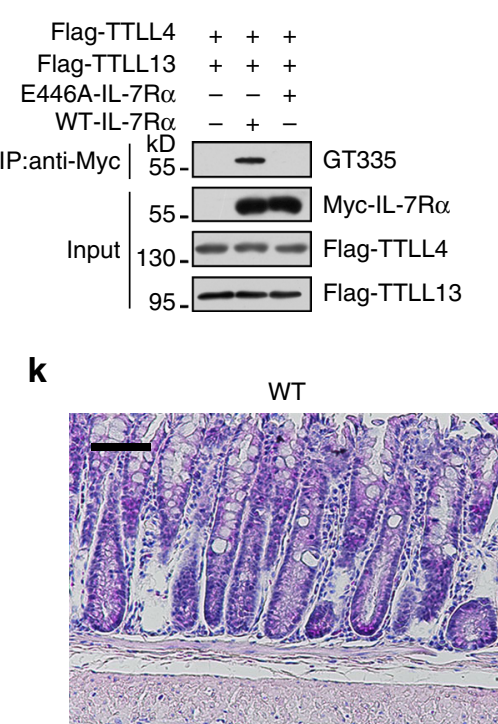

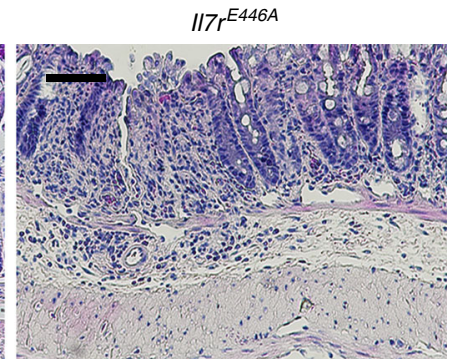


agonist $\mathrm{CoCl}_{2}$ abrogated the glutamylation of IL-7R $\alpha$ (Fig. 3h). Collectively, we conclude that IL-7R $\alpha$ is a novel substrate for CCP2.

IL-7R $\alpha$ is polyglutamylated at Glu446 by TTLL 4 and TTLL13. Nine polyglutamylases have been reported to catalyze protein glutamylation ${ }^{21,} 24$. To determine the physiological polyglutamylases catalyzing IL-7R $\alpha$ glutamylation, we examined expression patterns of all nine polyglutamylases in $\alpha \mathrm{LPs}$ and CHILPs of mouse BM through quantitative real-time PCR. We observed that Ttll4 and Ttll13 were highly expressed in $\alpha$ LPs and CHILPs, with peak expression in CHILPs (Fig. 4a). Additionally, Ttll4 and Ttll13 were highest expressed in CHILPs among all the hematopoietic progenitor cells (Fig. 4b). We next incubated recombinant intracellular segment rGST-IL-7R $\alpha$ with Flag-tagged TTLL4 or TTLL13 in vitro. We noticed that Flag-tagged TTLL4 and TTLL13 were able to precipitate rGST-IL-7R $\alpha$ (Fig. 4c). Their interactions were further verified by co-transfection assays (Fig. 4d). Moreover, IL-7R $\alpha$ was co-localized with TTLL4 and TTLL13 in CHILPs (Fig. 4e). We then conducted in vitro glutamylation assays by incubation of rGST-IL-7R $\alpha$ with Flag-TTLL4 or Flag-TTLL13. We found that rGST-IL-7R $\alpha$ was polyglutamylated by TTLL4 and TTLL13 (Fig. 4f). Importantly, TTLL4- and TTLL13-mediated polygutamylation of rGST-IL$7 \mathrm{R} \alpha$ was successfully removed by enzymatic active CCP2 (Fig. 4g). These data indicate that TTLL4 and TTLL13 are two polyglutamylases for IL-7R $\alpha$ polyglutamylation.

Glutamate-rich stretches and acidic environment at the acceptor sites have been reported to be important for glutamylation modification ${ }^{33}$. Based on the conservative aminoacid sequence analysis, only Glu446 and Glu447 were two conserved identical glutamic acid residues located on the loop region of intracellular domain of IL-7R $\alpha$ (Supplementary Fig. 2b), which might be potential acceptor site candidates for glutamylation. We then mutated Glu446 to Ala of IL-7R $\alpha$ (E446A-IL-7R $\alpha$ ) and incubated recombinant intracellular E446A-IL-7R $\alpha$ protein with Flag-TTLL4 or Flag-TTLL13 in vitro. We observed that E446A-IL-7R $\alpha$ mutant abolished TTLL4- or TTLL13-mediated glutamylation (Fig. 4h), suggesting IL-7R $\alpha$ is catalyzed by TTLL4 and TTLL13 at Glu446.

We next explored the physiological relevance of IL-7R $\alpha$ glutamylation in ILC3 differentiation. We silenced IL-7R $\alpha$ by LMP retrovirus-carried short hairpin RNA (shRNA) infection in CHILPs and then rescued expression of WT-IL-7R $\alpha$ or E446AIL-7R $\alpha$, followed by BM transplantation assays. Eight weeks after transplantation, IL-7R $\alpha$ knockdown with empty vector infection remarkably decreased ILC3 numbers (Supplementary Fig. 2c). By contrast, WT-IL-7R $\alpha$ restoration could rescue the normal number of ILC3s in recipient mice, whereas E446A-IL-7R $\alpha$ mutant overexpression had no such effect (Supplementary Fig. 2c). Parallelly, these observations were further validated by in vitro differentiation assays (Supplementary Fig. 2d, e).
Collectively, IL-7R $\alpha$ glutamylation is required for ILC3 development.

To further validate the authentic role of IL-7R $\alpha$ glutamylation in the regulation of ILC3 development, we generated E446A-IL7 R $\alpha$ mutation $\left(I l 7 r^{E 446 A}\right)$ mice through CRISPR/Cas9 technology. We noticed that IL-7R $\alpha$ really did not undergo glutamylation in CHILPs of $I l 7 r^{E 446 A}$ mice (Fig. 4i). Consistently, $I l 7 r^{E 446 A}$ mice displayed reduced numbers of ILC3s (Fig. $4 \mathrm{j}$ and Supplementary Fig. $2 \mathrm{f}$ ) and more severe intestinal injury post C. rodentium infection (Fig. 4k). Consequently, $I l 7 r^{E 446 A}$ mice died rapidly with C. rodentium challenge (Fig. 4l). Finally, we transplanted CD $45.2^{+}$ WT or $I l 7 r^{E 446 A} \mathrm{BM}$ cells into lethally irradiated CD $45.1^{+}$ recipients. Eight weeks after transplantation, $I l 7 r^{E 446 A} \mathrm{BM}$ transferred recipients displayed a reduced number of ILC3s compared to those of WT BM engraftment (Supplementary Fig. 2g). Collectively, we conclude that IL-7R $\alpha$ glutamylation is required for ILC3 development from CHILPs.

IL-7R $\alpha$ glutamylation promotes Sall3 expression by STAT5. IL-7R $\alpha$ pairs with the common $\gamma$-chain of IL-2R or TSLP receptor to detect IL-7 and TSLP, respectively, for the activation of STAT proteins in DCs, CD4 ${ }^{+} \mathrm{T}$ as well as B cells ${ }^{18,34,35}$. However, how IL-7R $\alpha$ glutamylation regulates the development of ILCs remains unclear. We then analyzed all STAT protein phosphorylation signals in $C c p 2^{+/+}$and $C c p 2^{-/-}$CHILPs. We found that only STAT5 was hyperphosphorylated in $C c p 2^{-1-}$ CHILPs compared to $C c p 2^{+/+}$CHILPs with IL-7 stimulation (Fig. 5a). However, other STAT proteins were not activated (Fig. 5a). We thus used STAT3 as a negative control in the following experiments. These observations were further validated by flow cytometry and immunofluorescence staining (Fig. 5b, c). These results indicate that IL-7R $\alpha$ glutamylation leads to STAT5 activation in CHILPs after IL-7 stimulation.

To further determine which TFs regulated IL-7R $\alpha$ glutamylation-mediated ILC3 development, we performed transcriptome microarray analysis of $C c p 2^{+/+}$vs. $C c p 2^{-/-}$CHILPs. Among top 10 upregulated TFs in $C c p 2^{-1-}$ CHILPs (Fig. 5d), we focused on Spalt-like transcription factor 3 (Sall3), which was a highest differentially expressed TF in $C c p 2^{-l-}$ CHILPs (Fig. 5e). Sall3, a member of the SAL family, is implicated in embryonic development and oncogenesis ${ }^{36,37}$. However, how Sall3 regulates the development of ILC3s is unknown. Intriguingly, the promoter region of Sall3 gene contained the STAT5-binding motif (TTCNNNGAA) ${ }^{38}$ (Supplementary Fig. 2h). Of note, anti-STAT5 antibody could immunoprecipitate Sall3 promoter by chromatin immunoprecipitation (ChIP) assay, whereas the anti-STAT5 antibody failed to immunoprecipitate other TF promoters (Fig. 5f). Consistently, the Sall3 promoter of Ccp $2^{-/-}$CHILPs bound to substantial STAT5 proteins compared to that of $C c p 2^{+/+}$CHILPs (Fig. $5 \mathrm{~g}$ ). The binding of STAT5 to Sall3 promoter was further verified by an EMSA assay (Fig. 5h). Consequently, STAT5 was able to activate Sall3 transcription

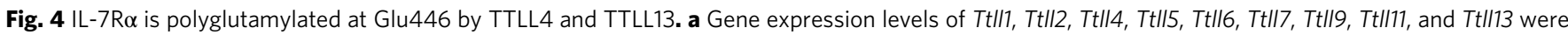
examined in $\alpha \mathrm{LPs}$ and CHILPs by real-time qPCR. Relative fold changes of gene expression values were normalized to endogenous Actb. $\mathbf{b}$ TtII4 and Tt/l13 expression patterns were analyzed in hematopoietic populations as in a. c rGST-IL-7R $\alpha$ bound to Flag-TTLL4 and Flag-TTLL13 by pulldown assay. d Myc-IL-7R $\alpha$ with Flag-TTLL4 or Flag-TTLL13 were co-transfected in 293 T cells for $36 \mathrm{~h}$, followed by immunoblotting assay. e CHILPs were incubated with antibodies against TTLL4, TTLL13, and IL-7R $\alpha$ by immunofluorescence staining. IL-7R $\alpha$, green; TTLL4 and TTLL13, red; nucleus, blue. Scale bar, $2 \mu$ m. f rGST-IL-7R $\alpha$ was incubated with TTLL4 and TTLL13 for in vitro glutamylation assay. $\mathbf{g}$ TTLL4- and TTLL13-mediated rGST-IL-7R $\alpha$ glutamylation was removed by CCP2. h Flag-TTLL4 and Flag-TTLL13 with Myc-IL-7R $\alpha$-wt or Myc-IL-7R $\alpha$-E446A were transfected into 293T cells. Cell lysates were immunoprecipitated by anti-Myc antibody and immunoblotted with indicated antibodies. $\mathbf{i}$ IL-7R $\alpha$ glutamylation was analyzed in BM cells from WT and $117 r^{E 446 A}$ by immunoblotting. $\mathbf{j}$ ILC3s were analyzed in WT and $\| 7 r^{E 446 A}$ mice by FACS. $n=6$ for each group. $\mathbf{k}$ Histology of colons from WT and $I / 7 r^{E 446 A}$ mice 8 days after infection with $C$. rodentium. Scale bars, $50 \mu \mathrm{m}$. I Survival rates were monitored at the indicated time points with $C$. rodentium infection. $n=8$ for each group. ${ }^{\star \star} P<0.01$ (Student's $t$-test). Data are representative of three independent experiments. Error bars in $\mathbf{a}, \mathbf{b}$, and $\mathbf{j}$ indicate s.d. 
a

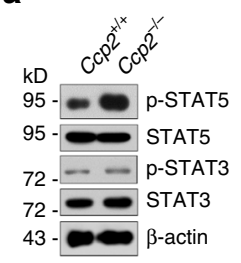

d

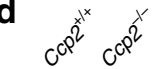

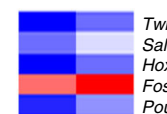
Sall3 Hoxc8
Fos 11 Fosl1
Pou4f1 Pou4t1
Foxh1 Foxh1
Mafb Creb3/1 Gsc Mixl1 Nab2 Hoxb4 Vax1 Zbtb3 Sec14/2 Eomes s/2 Olig1 Lmx1a Pmfbp1 Z 3300 Zfp52 Pparg Maged 1 Zfp39 Bach2 Pax5 Spic Spib Spib IIIIHigh

m

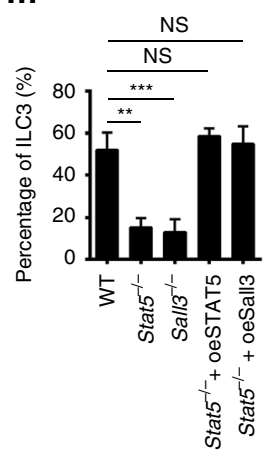

b
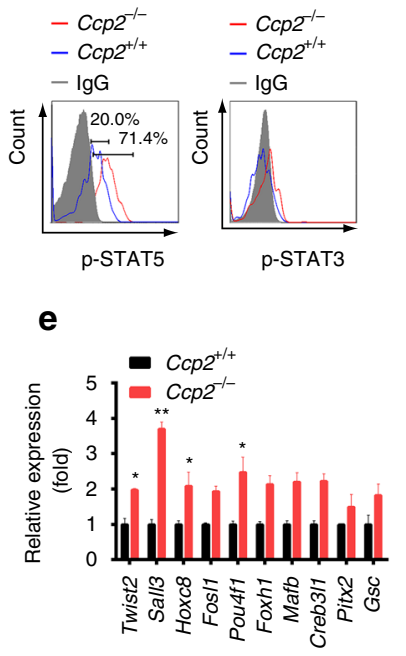
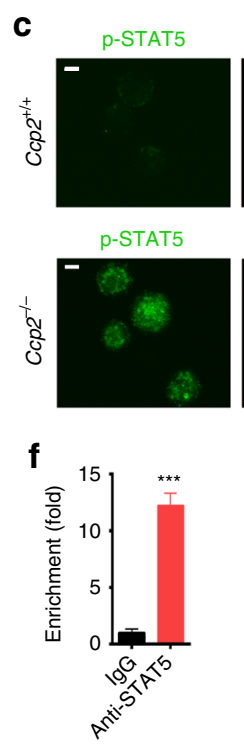

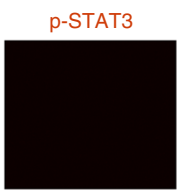

p-STAT3

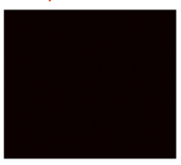

g

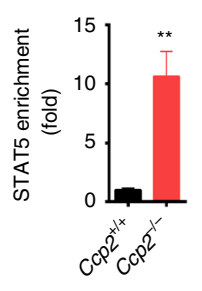

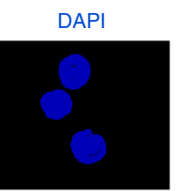

DAPI

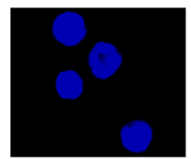

h Unlabeled probe $\quad-\quad-+$ Flag-STAT5 -+++

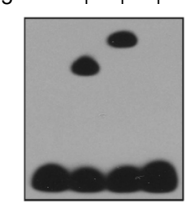

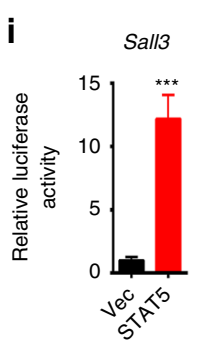

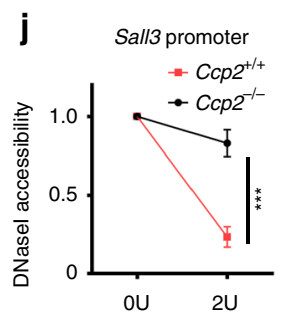

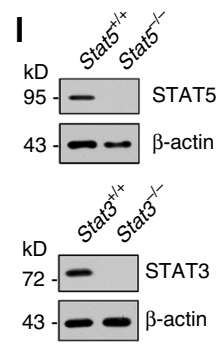

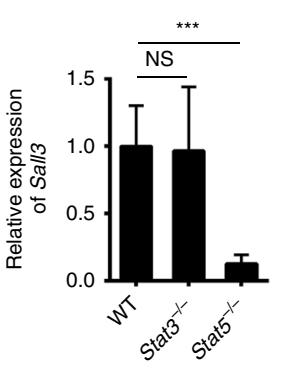

p
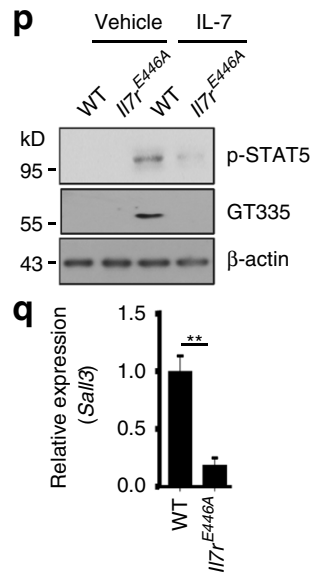

Fig. 5 IL-7R $\alpha$ glutamylation promotes Sall3 expression by STAT5. a-c Phosphorylation of STAT3 and STAT5 was tested by western blotting a, flow cytometry $\mathbf{b}$ and immunofluorescence staining $\mathbf{c}$ in CHILPs from WT and Ccp2 ${ }^{-/-}$mice after IL-7 stimulation. p-STAT5, green; p-STAT3, red; nucleus, blue.

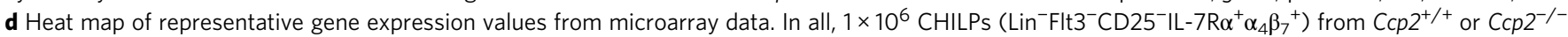
mice were sorted for microarray. e Analysis of indicated gene expression levels in $\mathrm{Ccp}^{+/+}$and $\mathrm{Ccp} 2^{-/-} \mathrm{CHILPs}$ by quantitative reverse transcription PCR (RT-qPCR). Relative fold changes of gene expression values were normalized to endogenous Actb. $\mathbf{f}, \mathbf{g}$ Enrichment assessment of STAT5 on Sall3 promoter in CHILPs from Ccp2 $2^{+/}$and $\mathrm{Ccp}^{-/-}$mice. $\mathbf{h}$ The association of STAT5 with Sall3 promoter was examined by EMSA. Sall3 promoter probe was biotin-labeled. i Flag-STAT5, pTK, and pGL3- Sall3 promoter were transfected into 293T cells for luciferase assay. $\mathbf{j}$ DNasel accessibility of Sall3 promoter in CHILPs from $\mathrm{Ccp}^{+/+}$and $\mathrm{Ccp} 2^{-/-}$mice was assessed. $\mathbf{k ~ H 3 K} 4 \mathrm{me} 3$ enrichment on Sall3 promoter was determined. CHILPs were isolated from Ccp2 ${ }^{+/+}$and $\mathrm{Ccp2^{-/- }}$ mice, followed by ChIP assay. I Sall3 mRNA levels were detected in WT, Stat $3^{-/-}$, and Stat $5^{-1-}$ CHILPs. m CHILPs were isolated from WT mice and cultured with mitomycin C-treated OP9 cells for in vitro differentiation assay. Stat5 $5^{-1}$ CHILPs were transfected with STAT5 or Sall3 overexpression plasmid. Percentages of ILC3s were analyzed by flow cytometry. oe overexpression. $\mathbf{n}$ ILC3s were analyzed in Sall $3^{+/+}$and Sall $3^{-/-}$mice. $n=6$ for each group. o Histology of colons from Sall $3^{+/+}$and Sall $3^{-/-}$mice 8 days after C. rodentium infection. Scale bars, $50 \mu \mathrm{m}$. p IL-7R $\alpha$ glutamylation and STAT5 phosphorylation in CHILPs in the absence or presence of IL-7. CHILPs from WT or II7rE446A BM were incubated with IL-7 and vehicle, followed by immunoblotting. $\beta$-actin was probed as loading controls. q Sall3 expression levels were tested in WT and II7rE446A CHILPs by RT-qPCR. Relative fold change of Sall3 expression values were normalized to endogenous Actb. ${ }^{\star \star} P<0.01,{ }^{\star \star \star} P<0.001$ (Student's $t$-test). Data represent three independent experiments. Error bars in $\mathbf{e}-\mathbf{g}$, $\mathbf{i}-\mathbf{n}$, and $\mathbf{q}$ indicate s.d. 
a

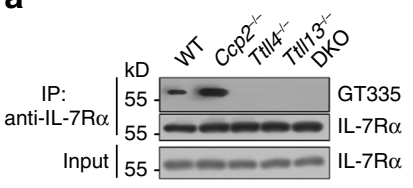

e
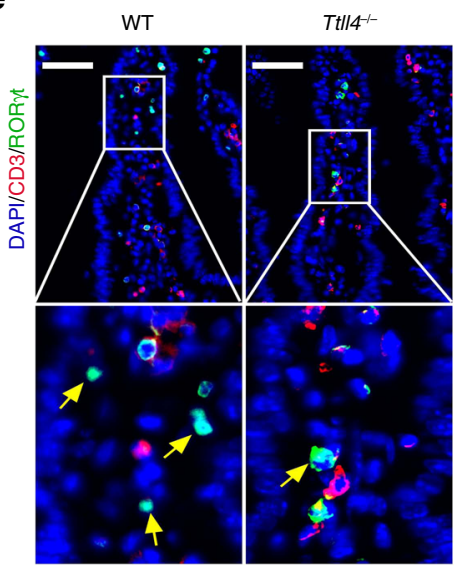

h

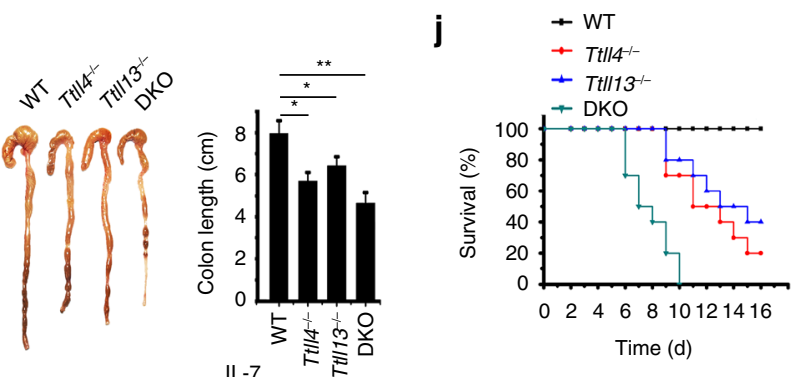

b
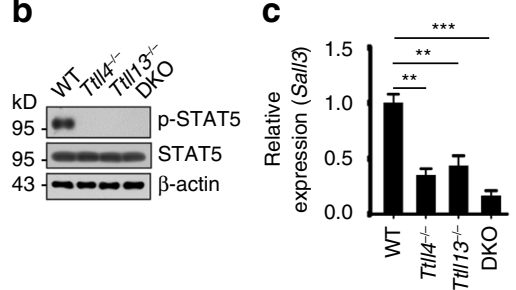

d

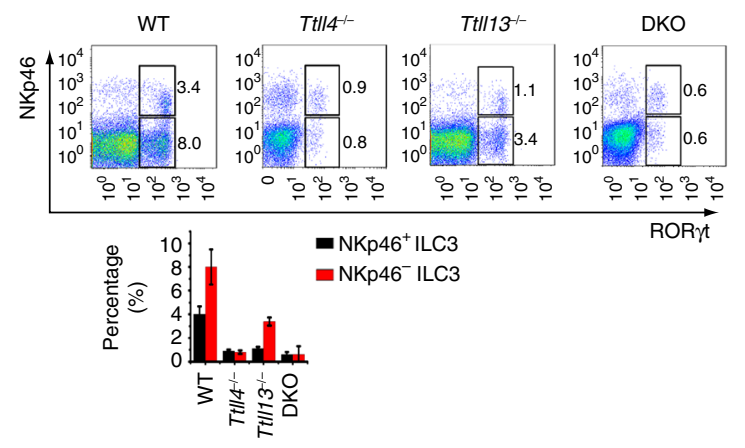

$\mathbf{f}$
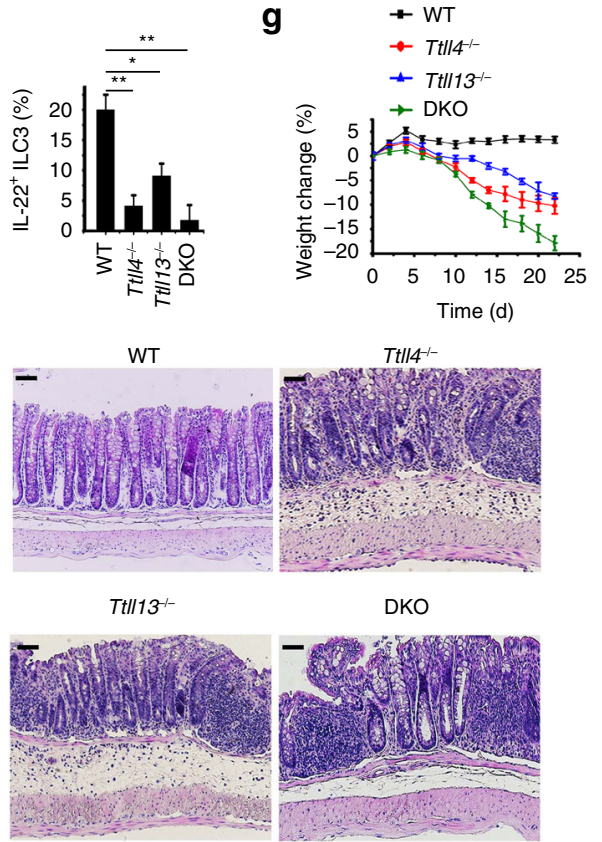

k

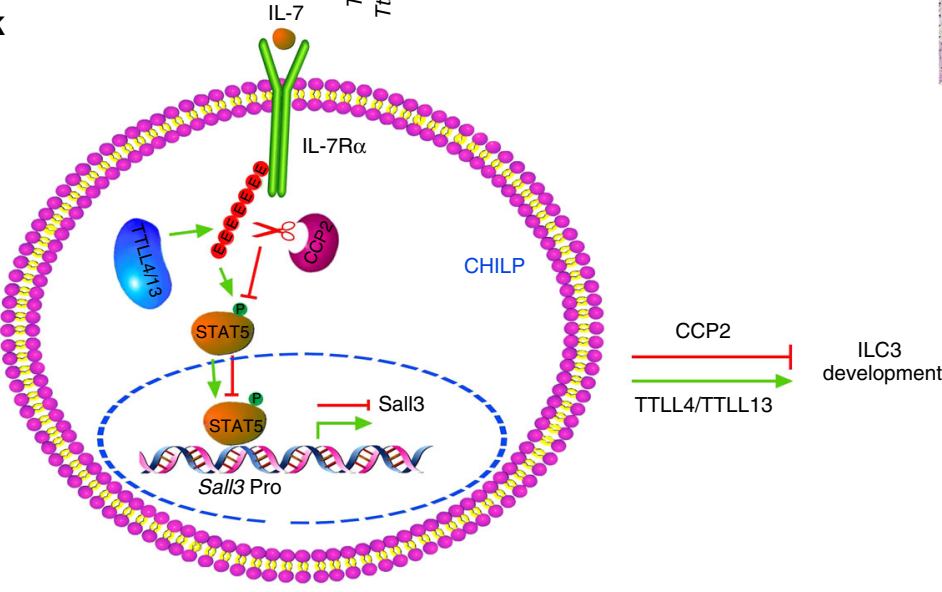

Fig. 6 Deletion of Tt/l4 or Tt/l13 impairs ILC3 development. a Detection of IL-7R $\alpha$ glutamylation in CHILPs by gating on $\mathrm{Lin}^{-} \mathrm{IL}-7 \mathrm{R} \alpha^{+} \mathrm{Flt} 3^{-} \mathrm{CD} 25^{-} \alpha_{4} \beta_{7}{ }^{+}$from

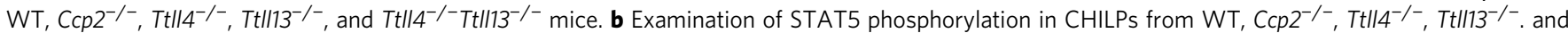

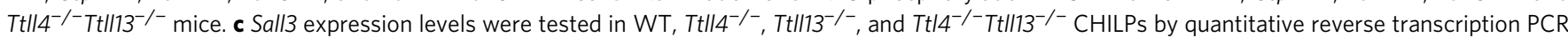
(RT-qPCR). Relative fold change of Sall3 expression values were normalized to endogenous Actb. d Flow cytometry analysis of ILC3s in small intestine lamina propria from WT, ttll $^{-/-}, \mathrm{Tt}_{1 / 13^{-/-}}$, and $\mathrm{Tt} / 14^{-/-} \mathrm{Tt} / 113^{-/-}$mice. Percentages of indicated cells were calculated and shown as means $\pm \mathrm{s} . \mathrm{d}$.

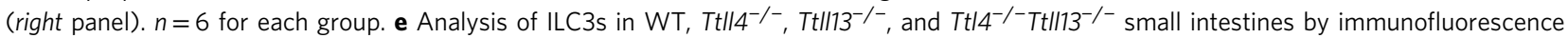
staining in situ. Arrowhead denotes ILC3 cells. Scale bars, $50 \mu \mathrm{m}$. f Analysis of IL-22 $2^{+}$ILC3s after IL23 stimulation by flow cytometry. $n=6$ for each group.

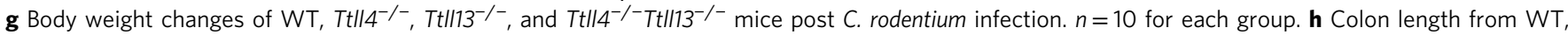

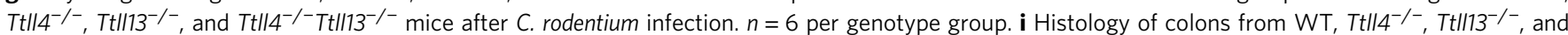

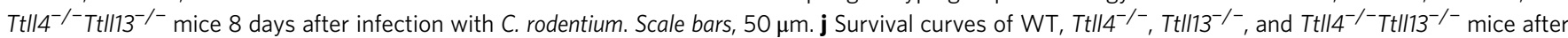
C. rodentium infection. $n=10$ for each group. In all, $2 \times 10^{9} \mathrm{C}$. rodentium was used by oral inoculation. $\mathbf{k}$ A working model represents glutamylation-mediated IL-7 signaling in the regulation of ILC3 development. Pro promoter. ${ }^{\star} P<0.05,{ }^{\star \star} P<0.01$, and ${ }^{\star \star \star} P<0.001$ (Student's $t$-test). Data represent three independent experiments. Error bars in $\mathbf{c}$, $\mathbf{d}$, and $\mathbf{f}-\mathbf{h}$ indicate s.d. 
through in vitro luciferase assays (Fig. 5i). Moreover, Sall3 promoter in $C c p 2^{-/-}$CHILPs was more accessible to DNase I digestion (Fig. 5j) and enriched more H3K4me3 (Fig. 5k), suggesting CCP2 deficiency promotes Sall3 expression.

We next generated Stat5 KO CHILPs via Cas9 knockin mice as described ${ }^{39}$ and Stat 3 conditional $\mathrm{KO}$ mice by crossing Stat $3^{\text {flox/flox }}$ mice to Mx1-Cre mice. STAT5 and STAT3 were successfully deleted in CHILPs (Fig. 5l). We noticed that Stat $5^{-1-}$ CHILPs abrogated Sall3 expression, while Stat3 $3^{-1-}$ CHILPs did not impact the expression of Sall3 (Fig. 5l). We then cultured CHILPs with OP9 cells in the presence of SCF and IL-7 in vitro. We found that Stat5 KO drastically reduced ILC3 numbers (Fig. $5 \mathrm{~m}$ ). However, rescue expression of STAT5 or Sall3 in Stat $^{-1-}$ CHILPs was able to restore ILC3 numbers comparable to WT mice (Fig. $5 \mathrm{~m}$ ), suggesting Sall 3 was a downstream target for STAT5 activation during ILC3 differentiation. We also generated Sall3 KO mice via Cas9 knockin mice. We observed that Sall3 KO mice also displayed decreased ILC3s and more susceptibility to C. rodentium infection compared with WT control mice (Fig. 5n, $o$ and Supplementary Fig. 2i). We next engrafted $5 \times 10^{4}$ CD $45.2^{+}$ LSKs from WT or Sall3 ${ }^{-1-}$ mice with $5 \times 10^{6} \mathrm{CD} 45.1^{+}$helper cells into lethally irradiated CD $45.1^{+}$recipients for 8 weeks, followed by analysis of mixed chimeras. We found that Sall ${ }^{-1-} \mathrm{BM}$ transferred recipients displayed a reduced number of ILC3s compared to those of WT BM engraftment (Supplementary Fig. 2j). More importantly, IL-7R $\alpha$ polyglutamylation indeed occurred in WT CHILPs with IL-7 stimulation, and STAT5 phosphorylation appeared as well (Fig. 5p). However, in the absence of IL-7, IL-7R $\alpha$ did not undergo polyglutamylation and no STAT5 phosphorylation appeared in WT CHILPs (Fig. 5p). By contrast, in the presence of IL-7, CHILPs from $I l 7 r^{E 446 \AA}$ mice did not undergo polyglutamylation and no STAT5 phosphorylation appeared either (Fig. 5p). Finally, $I l 7 r^{E 446 A}$ CHILPs did not activate the expression of Sall3 (Fig. 5q). Taken together, we conclude that IL-7R $\alpha$ glutamylation-mediated STAT5 activation initiates Sall3 expression that drives the development of ILC3s from CHILPs.

Deletion of Ttll4 or Ttll13 impairs ILC3 development. We next generated Ttll4 and Ttll13 KO mice via CRISPR/Cas9 technology (Supplementary Fig. 3a, b). We noticed that deletion of TTLL4 or TTLL13 abrogated IL-7R $\alpha$ glutamylation in CHILPs (Fig. 6a). Importantly, Ttll4 $4^{-1-}$ or Ttll13 $3^{-1-}$ CHILPs impaired STAT5 phosphorylation and blocked Sall3 expression in CHILPs (Fig. 6b, c). Consistently, Ttll4 or Ttll13 KO abolished ILC3 differentiation (Fig. 6d and Supplementary Fig. 3c). Ttll4 and Ttll13 double KO (DKO) almost suppressed the ILC3 formation in small intestines (Fig. 6d). These results were further confirmed by immunofluorescence staining (Fig. 6e). Additionally, Ttll4 or Ttll13 KO also dramatically reduced numbers of IL-22+ ILC3s (Fig. 6f). As expected, Ttll4 or Ttll13 KO remarkably declined ILCP generation (Supplementary Fig. 3d). These data suggest that TTLL4- and TTLL13-mediated IL-7R $\alpha$ glutamylation is required for the activation of Sall3 in CHILPs that drives the differentiation of ILC3s.

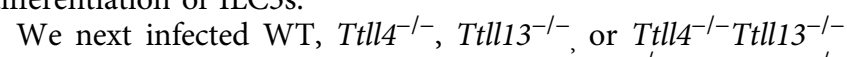
mice with $C$. rodentium. We observed that $T t l l 4^{-1-}$ and Ttll13 $3^{-/-}$ mice had higher bacterial loads in fecal, spleen, liver, and blood on day 8 post infection compared with their littermate WT control mice (Supplementary Fig. 3e-h). Additionally, Ttll4 ${ }^{-/-}$ and $T t l l 13^{-/}$mice lost their weights over $C$. rodentium infection (Fig. 6g), accompanied with shrinking length of colons (Fig. 6h). Following infection with $C$. rodentium, Ttll4 ${ }^{-1-}$ and Ttlll $^{-/-}$ mice displayed much more persistent intestinal damage, encompassing greater epithelial injury, crypt hyperplasia, and more infiltration of inflammatory cells, than those of their littermate WT control mice (Fig. 6i). Consistently, Ttll4 ${ }^{-/-}$and Ttll13 $3^{-/-}$ mice succumbed to bacterial infection (Fig. 6j). Expectedly, Ttll4 and Ttll13 DKO displayed much higher susceptibility to $C$. rodentium infection (Fig. $6 \mathrm{~g}-\mathrm{j}$ ). In sum, TTLL4- and TTLL13mediated IL-7R $\alpha$ glutamylation has a critical function in the differentiation and effector functions of ILC3s.

\section{Discussion}

ILCs are a distinct arm of the innate immune system, which can directly communicate with other hematopoietic and non-hematopoietic cells to regulate immunity, inflammation and tissue homeostasis ${ }^{1}$. However, how these ILC lineages develop and/or maintain remains unclear. In this study, we show that CCP2 deficiency causes increased numbers of ILC3s. With IL-7 engagement, IL-7R $\alpha$ undergoes polyglutamylation in CHILPs. IL-7R $\alpha$ polyglutamylation specifically activate STAT5 phosphorylation to initiate Sall3 expression for ILC3 development (Fig. 6k). In addition, Ttll4 ${ }^{-/-}$and Ttll13 $^{-/-}$mice abrogate IL-7R $\alpha$ polyglutamylation and Sall3 expression in CHILPs, leading to impaired ILC3 differentiation and more susceptibility to C. rodentium infection. Finally, E446A-IL-7R $\alpha$ mutation mice indeed abrogates Sall3 expression and ILC3 development.

The earliest progenitor cells specific to ILCs are CXCR6 ${ }^{+}$ integrin $\alpha_{4} \beta_{7}$-expressing CLPs, referred to as $\alpha$-lymphoid precursor $(\alpha \mathrm{LP})$ cells, which give rise to ILC1, ILC2, ILC3, and conventional NK cells $(\mathrm{cNK})^{40}$. The common progenitor to all ILC lineages (CHILP) is identified as its Lin $^{-}$IL-7R $\alpha^{+}$ Id $2^{+} \mathrm{CD} 25^{-} \alpha_{4} \beta_{7}^{+}$phenotype and differentiates to all ILC subsets, but not $\mathrm{cNKs}^{14}$. The common precursor to ILCs (ILCP) is defined by expression of TF PLZF and generates ILC1, ILC2, and ILC3 subpopulations ${ }^{15}$. In this study, we show that CCP2 is highly constitutively expressed in CHILPs and ILC3s, which blocks the deglutamylation of IL-7R $\alpha$ to drive ILC3 development. CCP2 deficiency causes increased numbers of ILC3s, but reduced numbers of ILC1s and ILC2s, which augments clearance of C. rodentium. Given that $\mathrm{CCP} 2$ is also moderately expressed in other cells such as $\mathrm{CD}^{+} \mathrm{T}$ cells, we thus cannot exclude the potential involvement of other cells in the bacterial clearance of CCP2 deficiency. Of note, CCP2 deficiency does not impact cell deaths of CHILPs and all ILC lineages. A recent study showed that different ILC subsets are defined by distinct gene-expression patterns ${ }^{41}$. Of note, cytokines such as IL-7, IL-15, and IL-2 play major roles in the regulation of ILC development. However, how CCP2-mediated IL-7 signaling regulates the switch balance of ILC development still needs to be further investigated. We notice that CCP members are differentially expressed in the hematopoietic progenitors and lineages we checked. We previously demonstrated that CCP6 is mostly highly expressed in BM and megakaryocytes, and also exhibits different expression profiles in different tissues and cell types ${ }^{28}$. Our findings suggest that different tissue and cell type distributions of CCPs may exert unique roles in the modulation of different physiological and pathological processes.

Protein polyglutamylation is catalyzed by a family of polyglutamylases, also called TTLLs ${ }^{24,25}$. The well-known substrates of polyglutamylation are tubulins and nucleosome assembly proteins ${ }^{33}$. Through regulating the interaction of microtubules (MTs) and MT-associated proteins (MAPs), polyglutamylation may exert major effects on MT-related cellular processes, including stability of centrosomes ${ }^{42}$, motility of cilia and flagella $^{43,44}$, neurite outgrowth ${ }^{45}$, as well as neurodegeneration ${ }^{26}$. A recent study delineates a structural MT recognition basis by catalysis with TTLL7 ${ }^{21}$. TTLLs have different expression patterns in diverse tissues and their functions are not entirely redundant ${ }^{43}$. 
We recently reported that TTLL4 and TTLL6 are most highly expressed in megakaryocytes ${ }^{28}$, both of whom catalyze polyglutamylation of Mad2 to modulate megakaryocyte maturation. Here we demonstrate that TTLL4 and TTLL13 are constitutively elevated in CHILPs, both of which can catalyze polyglutamylation of IL-7R $\alpha$ to regulate the development of ILC3s. Deletion of TTLL4 or TTLL13 impairs ILC3 differentiation and their effector functions. Thus, IL-7R $\alpha$ polyglutamylation mediated by TTLL4 or TTLL13 has a critical function in the regulation of ILC3 development from the stage of CHILPs.

IL-7R $\alpha$ (CD127), encoded by Il7r gene, forms a receptor complex with the common cytokine receptor $\gamma$-chain of IL-2R or TSLP receptor to sense IL-7 and TSLP, respectively ${ }^{18,46}$. The IL-7-IL-7R $\alpha$ ligand-receptor pair signaling is critical for proliferation and survival of $\mathrm{T}$ and $\mathrm{B}$ lymphocytes in a non-redundant fashion. Genetic aberrations of IL-7R $\alpha$ signaling lead to immune deficiency syndromes and other immune diseases $^{47}$, 48 . It has been reported that all ILC lineages express high levels of IL-7R $\alpha^{14}$. Of note, the ILCP CHILPs also express IL-7R $\alpha$, which gives rise to all ILCs. However, the molecular mechanism by which IL-7R $\alpha$ signaling regulates the development of ILCs remains elusive. In this study, we show that TTLL4 and TTLL13-mediated IL-7R $\alpha$ polyglutamylation regulates the differentiation of ILC3s from CHILPs. Mechanistically, polyglutamylated IL-7R $\alpha$ is able to activate STAT5 and phosphorylated STAT5 can directly bind to Sall3 promoter to initiate its transcription, which drives the development of ILC3s from CHILPs.

A CHILP cell has been defined that lacks expression of Flt3 and CD25 but expresses IL-7R $\alpha$ and $\alpha_{4} \beta_{7}{ }^{14}$. CHILPs differ from $\alpha$-LPs in that CHILPs express Id2. CHILPs generate all ILCs, including LTi cells, but they fail to give rise to conventional NK cells. Subsequently, their downstream precursor ILCPs (common precursor of ILCs), characterized by expression of the TF PLZF, lose the ability to generate LTi cells and produce all ILC1, ILC2, and ILC3 subsets ${ }^{15}$. ROR $\gamma t$ (encoded by Rorc) drives differentiation of ILC3s from their precursor ILCPs ${ }^{16}$. ROR $\gamma \mathrm{t}$ deletion causes a complete loss of ILC3s but not ILC1s or ILC2s. Runx 3 is also required for the development of ILC1s and ILC3s, but not for ILC2s $s^{6}$. GATA3 is also involved in the development of ILC3s, and It continues to exert a critical role in mature ILC3s ${ }^{49}$, 50. These observations suggest that the development of different ILC subsets are controlled by TF networks ${ }^{41}$. Sall3 (Spalt-like transcription factor 3) belongs to the SAL family, which is implicated in embryonic development ${ }^{36,37,51}$. However, how Sall3 regulates the development of ILCs is still unknown. Here we define that Sall3 is a downstream target of IL-7 signaling, whose expression induced by IL-7R $\alpha$ polyglutamylation drives CHILPs to differentiate ILC3s.

Glutamylation is highly conserved in all metazoans and protists, exerting critical roles in many physiological and pathological processes ${ }^{52}$. For example, TTLL7, the most abundantly expressed TTLLs in the mammalian nervous system, is conserved from acorn worm to primates, where it modulates neurite outgrowth and localization of dendritic $\mathrm{MAPs}^{45}$. ILC3s are enriched in Peyer's patches (PPs) and intestinal lamina propria ${ }^{30}$. Prior to the development of adaptive immunity, ILC3-induced IL-22 production has a critical function in priming innate immunity to eradicate C. rodentium ${ }^{53}$, 54. IL-22-deficient mice displayed exaggerated intestinal inflammation and impairment of the epithelial barrier and rapidly succumbed to bacterial infection. Given that ILC3s produce large amounts of IL-22, we thus used $C$. rodentium infection as a readout for determining the physiological function of ILC3s in the knockout mouse responses. The host protective effects of ILC3s are not restricted to bacterial infection in the intestine. ILC3s are also implicated in the resistance to infections of Candida albicans and Mycobacterium tuberculosis in the lungs ${ }^{55}, 56$. Thereby ILC3s may be targeted to enhance or block immune responses for inflammatory pathology and immunotherapy. In this study, we show that the glutamylation and deglutamylation of IL-7R $\alpha$ mediated by CCP2 and TTLL4/13 controls the development and effector function of ILC3s. Therefore, we strongly believe that it is necessary to develop specific inhibitors or agonists for these related polyglutamylases and CCPs. Manipulating polyglutamylation profiles by using these compounds, we may potentially target ILC3s for future clinical applications. In sum, IL-7R $\alpha$ polyglutamylation has a critical function in the regulation of ILC3 development and their effector function. Our findings provide new mechanistic insights into how polyglutamylation modulates ILC3 development.

\section{Methods}

Antibodies and reagents. Anti-CCP1 (LM-1A7), anti-CCP2 (S-13), anti-CCP3 (S-15), anti-CCP4 (T-17), anti-CCP5 (N-18), anti-CCP6 (N-14), anti-TTLL4 (S-14), anti-TTLL7 (E-12), anti-TTLL9 (C-20), anti-TTLL13 (D-16), anti-STAT4 (C-4), anti-P-STAT4 (E-2), anti-STAT6 (M-20), anti-P-STAT6 (sc-11762), anti-GST (6G9C6), and anti-Myc (9E10) were from Santa Cruz Biotechnology; Anti-STAT1 (14994), anti-P-STAT1 (9167), anti-STAT2 (72604), anti-STAT3 (9139), anti-P-STAT3 (9145), anti-H3K4me3 (9751), anti-STAT5 (9363), and anti-P-STAT5 (9351) were from Cell Signalling Technology (Danvers, USA). anti-IL-7R $\alpha$ (135004) was from Biolegend. The antibodies against P-STAT2 (SAB4503836), Sall3 (SAB2102075), Flag-tag (M1), $\beta$-actin (SP124), and His-tag (6AT18) were from Sigma-Aldrich (St Louis, USA). GT335 antibody (AG-20B-0020) was from AdipoGen. Antibodies against CD3 (17A2), CD19 (1D3), B220 (RA3-6B2), IL-7R $\alpha$ (A7R34), c-Kit (2B8), Sca-1 (D7), CD25 (PC61.5), CD11b (M1/70), CD11c (N418), Gr1 (RB6-8C5), F4/80 (BM8), Ter119 (TER-119), CD27 (LG.7F9), CD90 (HIS51), CD45.2 (104), ROR $\gamma$ t (AFKJS-9), NKp46 (29A1.4), CD244 (C9.1), Flt3 (A2F10), $\alpha_{4} \beta_{7}$ (DATK32), CD45.1 (A20), NK1.1 (PK136), IL-22 (IL22JOP), Thy1.2 (30-H12), and PLZF (Mags.21F7) were purchased from eBiosciences (San Diego, USA). Active caspase 3 antibody (550914) was purchased from BD Bioscience. All primary antibodies were used in a 1:2000 dilution for western blotting, in a 1:500 dilution for immunofluorescence staining and in a 1:100 dilution for flow cytometric staining. Paraformaldehyde (PFA, 158127), phenanthroline (Phen, 33510), CoCl2 (60818), and 4',6-diamidino-2-phenylindole (DAPI; (D9542)) were from Sigma-Aldrich. IL-22 ELISA kit (BMS6022) was purchased from eBiosciences.

Generation of knockout mice and II7rE446A mice. $C c p 1$ and $C c p 6$ knockout mice were described previously ${ }^{28}$. Ccp $2^{-1-}, C c p 3^{-1-}, C c p 4^{-1-}, C c p 5^{-1-}, T_{t l l} 4^{-1-}$, and Ttll13 $^{-/-}$mice were generated through CRISPR-Cas9 approaches as described ${ }^{29}$. Gt(ROSA)26Sor ${ }^{\text {tml(CAG-xstpx-cas9,-EGFP)Fezh }}, \operatorname{Rorc}(\gamma \mathrm{t})^{+/ G F P}$ and $I d 2^{+/ G F P}$ mice were purchased from the Jackson Laboratory. Stat $3^{\text {flox/llox }}$ was kindly provided by Dr Shizuo Akira (Osaka University, Japan). Stat $3^{\text {flox/flox; }} \mathrm{MxCre^{+ }}$ mice were obtained by crossing Stat $3^{\mathrm{f} / \mathrm{f}}$ mice with $\mathrm{MxCr} \mathrm{C}^{+}$mice. To induce STAT3 deletion, $200 \mu \mathrm{g}$ polyinosine-polycyticylic acid (poly(I:C)) was intraperitoneally injected to mice every other day for three times. Mouse experiments were performed according to the guidelines of the institutional animal care and use committees at the Institute of Biophysics, Chinese Academy of Sciences. For deletion of Sall3 in BM, B6;129-Gt(ROSA)26Sor ${ }^{\text {tml(CAG-xstpx-cas9,-EGFP)Fezh }}$ knockin mice were crossed with Vav-Cre transgenic mice to generate Rosa26-LSL-Cas $9^{+}$; Vav-Cre ${ }^{+}$ mice. In all, $2 \times 10^{6} \mathrm{BM}$ cells were infected with lentiCRISPRv2 containing sgSall 3 lentivirus. BM cells were then transplanted into lethally irradiated recipient mice $\left(\mathrm{CD} 45.1^{+}\right)$. Donor-derived ILC3s were analyzed 8 weeks post transplantation. Sall 3 deletion was confirmed by immunoblotting. For generation of $I l 7 r^{E 446 A}$ mice, the genome locus of $I l 7 r$ gene was knocked in with IL-7R $\alpha$-E446A mutation via a CRISPR-Cas9 approach. Mixture of Cas9 mRNA, single guide RNA (sgRNA), and IL-7R $\alpha-E 446$ A donor templates was microinjected into the cytoplasm of C57BL/6 fertilized eggs and transferred into the uterus of pseudopregnant ICR females. IL-7R $\alpha$-E446A mutations were identified by PCR screening and DNA sequencing. gRNA sequences are as follows: Ccp2: 5'-TAGAAATATTCTGGTTGATGTGG-3'; Ccp3: 5'-GGAGTATCAGCTAGGAAGATGGG-3'; Ccp4: 5'-AGCTCTGAGCTGGTGCTCCCAGG-3'; Ccp5: 5'-GGTTCTACTTCAGTGTCCGGGG-3'; Ttll4: 5'-TTTGCCTCACGTTGGTGCGGCGG-3'; Ttll13: 5'-TTTCTTGGCTACAACCGATAAGG-3'; Il7r: 5'-TTCTTCTTGATTCAGTACTGAGG-3'; Sall3: $5^{\prime}$-CCAGCATCTCAAGTCGGACG-3'. Mice used in all experiments were 8-weeks old. And we performed three independent experiments of each mouse from at least three mice for each group. The background of mice was C57BL/6, and mice were grouped by the same age and gender. Animal use and protocols were approved by the Institutional Animal Care and Use Committees at Institute of Biophysics, Chinese Academy of Sciences. 
Histology analysis. Mouse colons after C. rodentium infection were fixed in $4 \%$ PFA (Sigma-Aldrich) for $24 \mathrm{~h}$, washed twice with phosphate-buffered saline (PBS) and stored using 75\% ethanol before embedded in paraffin. Then colons in paraffin were sectioned and stained with hematoxylin and eosin (H\&E) according to standard laboratory procedures.

Intestinal lymphocyte separation. Protocols for lymphocyte isolation from the intestine had been described ${ }^{57}$. With some modifications, intestines were dissected and cleaned, then PPs were removed. Intestines were cut longitudinally and washed with Dulbecco's Phosphate Buffered Saline (dPBS) five times. Then intestines were cut into pieces, and washed with solution I buffer (10 mM HEPES and $5 \mathrm{mM}$ EDTA in Hank's Balanced Salt Solution (HBSS)) five times. For LPL isolation, the intestinal fragments were digested with solution II buffer containing DNaseI, 5\% FBS, $0.2 \mathrm{mg} / \mathrm{ml}$ collagenase II and collagenase III there times at $37^{\circ} \mathrm{C}$. Then the tissues were sifted through $70-\mu \mathrm{m}$ strainers.

Flow cytometry. BM cells were flushed out from femurs in PBS buffer and sifted through $70-\mu \mathrm{m}$ cell strainers. For BM flow cytometric analysis, CLP

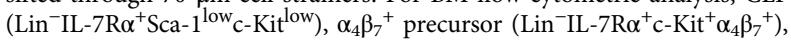
CHILP (Lin ${ }^{-}$IL-7R $\alpha^{+} \mathrm{Flt3}^{-} \mathrm{CD} 25^{-} \mathrm{Id} 2^{+} \alpha_{4} \beta_{7}{ }^{+}$or lin ${ }^{-} \mathrm{IL}-7 \mathrm{R} \alpha^{+} \mathrm{Flt} 3^{-} \mathrm{CD} 25^{-} \alpha_{4} \beta_{7}{ }^{+}$ $\mathrm{CD} 27^{+} \mathrm{CD} 244^{+}$), ILCP $\left(\mathrm{Lin}^{-} \mathrm{IL}-7 \mathrm{R} \alpha^{+} \mathrm{Id} 2^{+} \alpha_{4} \beta_{7}{ }^{+} \mathrm{PLZF}^{+}\right.$), ILC3 $\left(\mathrm{Lin}^{-} \mathrm{ROR} \gamma \mathrm{t}^{+} \mathrm{CD} 45^{+}\right.$), and IL-22 ${ }^{+}$ILC3 $\left(\mathrm{Lin}^{-} \mathrm{CD} 45^{+} \mathrm{IL}-22^{+}\right.$) populations were analyzed or sorted with a FACSAria III instrument (BD Biosciences). Lineage cocktail antibody contains anti-B220, anti-CD3, anti-Ter119, anti-Gr1, anti-CD11b, anti-CD19, and anti-NK1.1. Data were analyzed using the FlowJo 7.6.1 software.

Immunofluorescence assay. Cells were isolated by fluorescence-activated cell sorting (FACS) and fixed with 4\% PFA for $20 \mathrm{~min}$ at room temperature, then followed by $0.5 \%$ NP40 permeabilization and $10 \%$ donkey serum blocking. Cells were incubated with antibodies at $4{ }^{\circ} \mathrm{C}$ overnight, and then incubated with fluorescence-conjugated secondary antibodies. DAPI was used for nucleus staining. Cells were visualized by laser scanning confocal microscopy (Olympus FV1000, Olympus, Japan).

Western blot. Cells were lysed with RIPA buffer $(150 \mathrm{mM} \mathrm{NaCl}, 0.5 \%$ sodium deoxycholate, $0.1 \%$ SDS, $1 \%$ NP40, 1 mM EDTA, $50 \mathrm{mM}$ Tris ( $\mathrm{pH} 8.0$ )), followed by separation with SDS-PAGE. Samples were then transferred to NC membrane and incubated with primary antibody in 5\% bovine serum albumin (BSA). After washing with Tris Buffered Saline, with Tween-20 (TBST) three times, membranes were incubated with horseradish peroxidase (HRP)-conjugated secondary antibodies. At least $1 \times 10^{5}$ cells were used for a single sample for whole cell blots. See Supplementary Figs. 4 and 5 for uncropped blots.

Quantitative real-time PCR. Cell populations were isolated by FACS. And total RNAs were extracted with an RNA Miniprep Kit (Tiangen, Beijing, China) according to the manufacturer's protocol. Then complementary DNA (cDNA) was synthesized with the M-MLV reverse transcriptase (Promega, Madison, USA). mRNA transcripts were analyzed with ABI 7300 quantitative PCR (qPCR) system using specific primer pairs. Relative expressions were calculated and normalized to $A c t b$ expression. Primers for TTLLs and CCPs were described ${ }^{28}$. Other specific primers are as follows: Il22 forward, 5'-TTGTGCGATCTCTGATGGCT-3'; Il22 reverse, 5'-CCAGCATAAAGGTGCGGTTG-3'; Twist 2 forward: 5'-CAGAGCGACGAGATGGACAA-3'; reverse: 5'-GAGAAGG CGTAGCTGAGACG-3'; Sall3 forward: $5^{\prime}$-CCTGATTCTTCCTGGTGGAGT-3'; reverse: 5' -CTCTGGAAAA CGCCACAGAC-3'; Hoxc8 forward: 5' -ACAGTAGCGAAG GACAAGGC-3'; reverse: $5^{\prime}$-CTTCAATCCGGCGCTTTCTG-3'; Fos 1 1 forward: 5'-CCGGTCCA CAGAGGTTCATC-3'; reverse: 5'-CTGGGCTGGATGTTCGGTAG-3'; Pou $4 f$ forward: 5'-GCACACTGGGGAGCTGAG-3'; reverse: 5'-AAACGAACA AGGTGGGAGGG-3'; Gapdh forward: 5'-TGCACCACCAACTGCTTAG-3'; reverse: 5'-GGATGCAGGGATGATGTTC-3'.

Chromatin immunoprecipitation assay. CHILPs $\left(1 \times 10^{5}\right)$ were isolated by FACS gating on $\mathrm{Lin}^{-} \mathrm{IL}-7 \mathrm{R} \alpha^{+} \mathrm{Flt} 3^{-} \mathrm{CD} 25^{-} \alpha_{4} \beta_{7}{ }^{+}$and cross-linked with $1 \%$ formaldehyde at $37^{\circ} \mathrm{C}$ for $10 \mathrm{~min}$. Then cells were washed twice with PBS, lysed and sonicated to get 300 to $500 \mathrm{bp}$ DNA fragments. Lysates were incubated with $4 \mu \mathrm{g}$ antibody overnight at $4{ }^{\circ} \mathrm{C}$. Salmon sperm DNA/protein agarose beads were added for DNA immunoprecipitation. After washing, DNA was eluted from beads and purified. DNA fragments were analyzed using qPCR. Primers used for ChIP were as follows: Sall3 forward: 5'-TAGTTCTCTTGCGCTCTTCCC-3', reverse: 5'-CTTGGAA AGCTGTCTTTCGGT-3'.

Recombinant protein preparation. cDNAs were cloned from a BM cDNA library. $\mathrm{CCP} 2$ was subcloned into $\mathrm{H}-\mathrm{MBP}-3 \mathrm{c}$ for MBP-tagged protein expression vectors. IL-7R $\alpha$ was cloned into pGEX-6p-1 plasmid for GST-tagged protein expression. Plasmids were transformed into E. coli strain BL21 (DE3), followed by induction with $0.2 \mathrm{mM}$ isopropyl- $\beta$-D-thiogalactoside (IPTG) at $16^{\circ} \mathrm{C}$ for $24 \mathrm{~h}$. Cells were collected and lysed by supersonic, followed by purification through Amylose or GST resins.
EMSA assay. EMSA experiments were conducted according to the manufacturer's protocol with a Light Shift Chemiluminescent RNA EMSA Kit (Thermo Scientific). Briefly, Flag-STAT5 was incubated with or without unlabeled probe for competitive reaction and anti-STAT5 antibody for super shift at room temperature for $20 \mathrm{~min}$ in a reaction buffer. Then, Biotin-labeled probe was added into the reaction system and incubated for $20 \mathrm{~min}$ at room temperature. Samples were carried out in $4 \%$ polyacrylamide gel in $0.5 \times$ TBE buffer. After transferred on a nylon membrane (Amersham Biosciences), the labeled DNA was cross-linked by ultraviolet, probed with streptavidin-HRP conjugate and then incubated with the detection substrate The probe sequence for Sall 3 was: 5'-CGGAGCCTAAAGCTGTTGCTTCGTGGAACTTAGA CTAGCGGGAGAATTCAGTGTG-3'.

DNase I accessibility assay. DNaseI digestion assay has been described previously ${ }^{28}$. In brief, Nuclei were purified from CHILPs according to the manufacturer's protocol with the Nuclei isolating Kit (Sigma-Aldrich). Then nuclei were resuspended with DNase I digestion buffer and treated with indicated units of DNase I (Sigma, USA) at $37^{\circ} \mathrm{C}$ for $5 \mathrm{~min}$. In all, $2 \times$ DNase I stop buffer (20 mM Tris Ph 8.0, 4 mM EDTA, 2 mM EGTA) was added to stop reactions. DNA was extracted and examined by qPCR.

BM transplantation. In all, $5 \times 10^{4} \mathrm{CD} 45.2^{+}$LSK from $C c p 2^{+/+}$or $C c p 2^{-1-}$ mice with $5 \times 10^{6} \mathrm{CD} 45.1^{+}$helper cells were transplanted into lethally irradiated CD $45.1^{+}$recipients. Eight weeks after transplantation, percentages of ILC3s derived from donor cells were analyzed by FACS. For competitive transplantation, $1 \times 10^{6}$ CD 45.2 $2^{+}$BM cells and $1 \times 10^{6}$ CD $45.1^{+}$BM cells were injected into lethally irradiated CD $45.1^{+}$-recipient mice. Eight weeks after transplantation, ratios of CD 45.2 $2^{+}$ILC3 to CD45.1 $1^{+}$ILC3 were analyzed. For IL-7R $\alpha$ knockdown or overexpression, $1 \times 10^{5}$ CHILP cells were infected with retrovirus carrying shRNAs or overexpression sequences, followed by BM transplantation. Eight weeks after transplantation, chimeras were analyzed by FACS. Small interfering RNA sequences against IL-7R $\alpha$ were cloned into LMP plasmid and IL-7R $\alpha$ was cloned into $\mathrm{pMY}$ vector.

Gene deletion in CHILPs by CRISPR/Cas 9 technology. Stat 5 and Sall3 deletion in CHILPs were generated using Cas 9 knockin mice according to the standard protocol provided by Zhang's lab ${ }^{39}$. Briefly, sgRNA was generated by online CRISPR Design Tool (http://tools.genome-engineering.org) and cloned into lentiCRISPRv2 for lentivirus production in $293 \mathrm{~T}$ cells. Then CHILPs were infected with lentivirus for Stat 5 or Sall3 deletion. sgRNA sequences of Stat 5 were: Stat5a: 5'-AGGTAGTGCCGGACCTCGAT-3'; Stat5b: 5'-AAATAATGTCGCAC CTCGAT-3'.

RNA interference. Sequences for RNA interference were designed according to MSCV-LTRmiR30-PIG (LMP) system instructions. LMP vectors containing target sequences were constructed. The target sequence against IL-7R $\alpha$ : 5' - GCGTA TGTCACCATGTCTAGT-3'

In vitro ILC differentiation assay. In vitro ILC differentiation assay was described previously ${ }^{15}$. In brief, CHILPs were sorted and cultured for 14 days on mitomycin C-treated OP9 feeder cells supplemented with IL-7 (25 ng/ml, Cat\#: 217-17) and rSCF (25 ng/ml, Cat\#: 250-03). Then cells were collected for flow cytometry.

C. rodentium infection. WT, Ccp $2^{-/-}, C c p 6^{-/-}, T t l l 4^{-/-}, T t l l 13^{-/-}$, and Ttll $^{-l-}{\text { Ttll } 13^{-/-} \text {mice were infected with } 5 \times 10^{9} \text { C. rodentium orally as described }}^{6}$ C. rodentium was a gift from Dr Baoxue Ge (Shanghai Institutes for Biological Sciences, Chinese Academy of Sciences). Mice were sacrificed by cervical dislocation to examine colon pathology and bacterial loads on day 8 post infection. Fecals, spleens, livers, blood, small intestines, and colons were collected from infected mice on day 8 after infection. Fecals, spleens, and livers were weighed and homogenized, and homogenates were plated on MacConkey agar plates for analysis of bacterial counts. Lamina propria lymphocytes (LPLs) were isolated from small intestines of infected mice, followed by analysis of ILC3s and IL-22.

In vitro glutamylation assay. Detailed protocol for in vitro glutamylation assay was described as previously described ${ }^{28}$. In brief, CCP2, TTLL4, and TTLL13 were transfected into $293 \mathrm{~T}$ cells for $48 \mathrm{~h}$. Cells were harvested and lysed. Supernatants were incubated with GST-IL-7R $\alpha$ at $37^{\circ} \mathrm{C}$ for $2 \mathrm{~h}$. GST-IL-7R $\alpha$ was precipitated and tested for glutamylation with GT335 antibody.

Statistical analysis. An unpaired Student's $t$-test was used as statistical analysis in this study. Statistical calculation was performed by using Microsoft Excel or SPSS 13

Data availability. All data generated or analyzed during this study are included in this published article and its Supplementary Information Files. Microarray data, are deposited in the Genebank as GSE97487. 
Received: 4 January 2017 Accepted: 13 June 2017

Published online: 10 August 2017

\section{References}

1. Artis, D. \& Spits, H. The biology of innate lymphoid cells. Nature 517, 293-301 (2015).

2. De Obaldia, M. E. \& Bhandoola, A. Transcriptional regulation of innate and adaptive lymphocyte lineages. Annu. Rev. Immunol. 33, 607-642 (2015).

3. Takatori, H. et al. Lymphoid tissue inducer-like cells are an innate source of IL17 and IL-22. J. Exp. Med. 206, 35-41 (2009).

4. Buonocore, S. et al. Innate lymphoid cells drive interleukin-23-dependent innate intestinal pathology. Nature 464, 1371-1375 (2010).

5. Chang, Y. J. et al. Innate lymphoid cells mediate influenza-induced airway hyper-reactivity independently of adaptive immunity. Nat. Immunol. 12, 631-638 (2011).

6. Ebihara, T. et al. Runx 3 specifies lineage commitment of innate lymphoid cells. Nat. Immunol. 16, 1124-1133 (2015).

7. Bernink, J. H. et al. Interleukin-12 and -23 control plasticity of CD127(+) group 1 and group 3 innate lymphoid cells in the intestinal lamina propria. Immunity 43, 146-160 (2015).

8. Seehus, C. R. et al. The development of innate lymphoid cells requires TOXdependent generation of a common innate lymphoid cell progenitor. Nat. Immunol. 16, 599-608 (2015).

9. Brestoff, J. R. et al. Group 2 innate lymphoid cells promote beiging of white adipose tissue and limit obesity. Nature 519, 242-246 (2015).

10. Eberl, G. et al. An essential function for the nuclear receptor RORgamma(t) in the generation of fetal lymphoid tissue inducer cells. Nat. Immunol. 5, 64-73 (2004)

11. Guo, X. et al. Innate lymphoid cells control early colonization resistance against intestinal pathogens through ID2-dependent regulation of the microbiota. Immunity 42, 731-743 (2015).

12. Guo, X. et al. Induction of innate lymphoid cell-derived interleukin-22 by the transcription factor STAT3 mediates protection against intestinal infection. Immunity 40, 25-39 (2014)

13. Diefenbach, A., Colonna, M. \& Koyasu, S. Development, differentiation, and diversity of innate lymphoid cells. Immunity 41, 354-365 (2014).

14. Klose, C. S. et al. Differentiation of type 1 ILCs from a common progenitor to all helper-like innate lymphoid cell lineages. Cell 157, 340-356 (2014).

15. Constantinides, M. G., McDonald, B. D., Verhoef, P. A. \& Bendelac, A. A committed precursor to innate lymphoid cells. Nature 508, 397-401 (2014).

16. Montaldo, E. et al. Human RORgammat $(+) \mathrm{CD} 34(+)$ cells are lineage-specified progenitors of group 3 RORgammat(+) innate lymphoid cells. Immunity 41, 988-1000 (2014).

17. Sanos, S. L. et al. RORgammat and commensal microflora are required for the differentiation of mucosal interleukin 22-producing NKp46+cells. Nat. Immunol. 10, 83-91 (2009).

18. Ziegler, S. F. \& Artis, D. Sensing the outside world: TSLP regulates barrier immunity. Nat. Immunol. 11, 289-293 (2010).

19. Luca, V. C. et al. Structural biology. Structural basis for Notch1 engagement of Delta-like 4. Science 347, 847-853 (2015).

20. Fang, L. et al. A methylation-phosphorylation switch determines Sox2 stability and function in ESC maintenance or differentiation. Mol. Cell 55, 537-551 (2014).

21. Garnham, C. P. et al. Multivalent microtubule recognition by tubulin tyrosine ligase-like family glutamylases. Cell 161, 1112-1123 (2015).

22. Edde, B. et al. Posttranslational glutamylation of alpha-tubulin. Science 247, 83-85 (1990)

23. Valenstein, M. L. \& Roll-Mecak, A. Graded control of microtubule severing by tubulin glutamylation. Cell 164, 911-921 (2016).

24. van Dijk, J. et al. A targeted multienzyme mechanism for selective microtubule polyglutamylation. Mol. Cell 26, 437-448 (2007).

25. Janke, C. et al. Tubulin polyglutamylase enzymes are members of the TTL domain protein family. Science 308, 1758-1762 (2005).

26. Rogowski, K. et al. A family of protein-deglutamylating enzymes associated with neurodegeneration. Cell 143, 564-578 (2010).

27. Fernandez-Gonzalez, A. et al. Purkinje cell degeneration (pcd) phenotypes caused by mutations in the axotomy-induced gene, Nnal. Science $\mathbf{2 9 5}$, 1904-1906 (2002).

28. Ye, B. et al. Cytosolic carboxypeptidase CCP6 is required for megakaryopoiesis by modulating Mad2 polyglutamylation. J. Exp. Med. 211, 2439-2454 (2014).
29. Xia, P. et al. Glutamylation of the DNA sensor cGAS regulates its binding and synthase activity in antiviral immunity. Nat. Immunol. 17, 369-378 (2016).

30. Serafini, N. et al. Gata3 drives development of RORgammat+group 3 innate lymphoid cells. J. Exp. Med. 211, 199-208 (2014).

31. Klose, C. S. et al. A T-bet gradient controls the fate and function of CCR6RORgammat+innate lymphoid cells. Nature 494, 261-265 (2013).

32. Berezniuk, I. et al. Cytosolic carboxypeptidase 1 is involved in processing alphaand beta-tubulin. J. Biol. Chem. 287, 6503-6517 (2012)

33. van Dijk, J. et al. Polyglutamylation is a post-translational modification with a broad range of substrates. J. Biol. Chem. 283, 3915-3922 (2008).

34. Miller, M. L. et al. Basal NF-kappaB controls IL-7 responsiveness of quiescent naive T cells. Proc. Natl Acad. Sci. USA 111, 7397-7402 (2014).

35. Patton, D. T., Plumb, A. W. \& Abraham, N. The survival and differentiation of pro-B and pre-B cells in the bone marrow is dependent on IL-7Ralpha Tyr449. J. Immunol. 193, 3446-3455 (2014).

36. Parrish, M. et al. Loss of the Sall3 gene leads to palate deficiency, abnormalities in cranial nerves, and perinatal lethality. Mol. Cell Biol. 24, 7102-7112 (2004).

37. Love, C. et al. The genetic landscape of mutations in Burkitt lymphoma. Nat. Genet. 44, 1321-1325 (2012).

38. Ihle, J. N. STATs: signal transducers and activators of transcription. Cell 84, 331-334 (1996).

39. Platt, R. J. et al. CRISPR-Cas9 knockin mice for genome editing and cancer modeling. Cell 159, 440-455 (2014)

40. Cherrier, M., Sawa, S. \& Eberl, G. Notch, Id2, and RORgammat sequentially orchestrate the fetal development of lymphoid tissue inducer cells. J. Exp. Med. 209, 729-740 (2012).

41. Robinette, M. L. et al. Transcriptional programs define molecular characteristics of innate lymphoid cell classes and subsets. Nat. Immunol. 16, 306-317 (2015).

42. Bobinnec, Y. et al. Centriole disassembly in vivo and its effect on centrosome structure and function in vertebrate cells. J. Cell Biol. 143, 1575-1589 (1998).

43. Bosch Grau, M. et al. Tubulin glycylases and glutamylases have distinct functions in stabilization and motility of ependymal cilia. J. Cell Biol. 202, 441-451 (2013).

44. Suryavanshi, S. et al. Tubulin glutamylation regulates ciliary motility by altering inner dynein arm activity. Curr. Biol. 20, 435-440 (2010).

45. Ikegami, K. et al. TTLL7 is a mammalian beta-tubulin polyglutamylase required for growth of MAP2-positive neurites. J. Biol. Chem. 281, 30707-30716 (2006).

46. Corcoran, A. E., Riddell, A., Krooshoop, D. \& Venkitaraman, A. R. Impaired immunoglobulin gene rearrangement in mice lacking the IL-7 receptor. Nature 391, 904-907 (1998).

47. Lundmark, F. et al. Variation in interleukin 7 receptor alpha chain (IL7R) influences risk of multiple sclerosis. Nat. Genet. 39, 1108-1113 (2007).

48. Ye, S. K. et al. Induction of germline transcription in the TCRgamma locus by Stat5: implications for accessibility control by the IL-7 receptor. Immunity $\mathbf{1 1}$ 213-223 (1999).

49. Yagi, R. et al. The transcription factor GATA3 is critical for the development of all IL-7Ralpha-expressing innate lymphoid cells. Immunity 40, 378-388 (2014).

50. Zhong, C. et al. Group 3 innate lymphoid cells continuously require the transcription factor GATA-3 after commitment. Nat. Immunol. 17, 169-178 (2016).

51. Wei, X. et al. Aberrant hypermethylation of SALL3 with HPV involvement contributes to the carcinogenesis of cervical cancer. PLOS ONE 10, e0145700 (2015).

52. Garnham, C. P. \& Roll-Mecak, A. The chemical complexity of cellular microtubules: tubulin post-translational modification enzymes and their roles in tuning microtubule functions. Cytoskeleton 69 442-463 (2012)

53. Sonnenberg, G. F., Fouser, L. A. \& Artis, D. Border patrol: regulation of immunity, inflammation and tissue homeostasis at barrier surfaces by IL-22. Nat. Immunol. 12, 383-390 (2011).

54. Satoh-Takayama, N. et al. Microbial flora drives interleukin 22 production in intestinal NKp46+cells that provide innate mucosal immune defense. Immunity 29, 958-970 (2008).

55. Gladiator, A., Wangler, N., Trautwein-Weidner, K. \& LeibundGut-Landmann, S. Cutting edge: IL-17-secreting innate lymphoid cells are essential for host defense against fungal infection. J. Immunol. 190, 521-525 (2013).

56. Pitt, J. M. et al. Blockade of IL-10 signaling during bacillus Calmette-Guerin vaccination enhances and sustains Th1, Th17, and innate lymphoid IFNgamma and IL-17 responses and increases protection to Mycobacterium tuberculosis infection. J. Immunol. 189, 4079-4087 (2012). 
57. Lee, J. S. et al. AHR drives the development of gut ILC22 cells and postnatal lymphoid tissues via pathways dependent on and independent of Notch. Nat. Immunol. 13, 144-151 (2012).

\section{Acknowledgements}

We thank Dr Dan Littman for providing pMIGR plasmid. We thank J.Li (Cnkingbio Company Ltd, Beijing, China) for technical support. We thank Jing Cheng, Jianhua Wang, Junfeng Hao, Yan Teng, Dongdong Fan, and Junying Jia for technical support. We thank Xiang Shi and Liangming Yao for technical help and assistance with animal procedures. This work was supported by the National Natural Science Foundation of China (31530093, 91640203, 31429001, 31670886, 31470864, 81572433, 81572433, 31601189,81672956 , and 81472413), the Strategic Priority Research Programs of the Chinese Academy of Sciences (XDB19030203, XDA12020219).

\section{Author contributions}

B.L. designed and performed experiments, analyzed data, and wrote the paper; B.Y. performed experiments and analyzed data; G.H. performed some experiments; L.Y. crossed some mice; P.Z., J.W., and Y.D. analyzed data. X.Z., S.M., and T.Y. build up animal models and analyzed data; Z.F. initiated the study, organized, designed, and wrote the paper.

\section{Additional information}

Supplementary Information accompanies this paper at doi:10.1038/s41467-017-00235-X.
Competing interests: The authors declare no competing financial interests.

Reprints and permission information is available online at http://npg.nature.com/ reprintsandpermissions/

Publisher's note: Springer Nature remains neutral with regard to jurisdictional claims in published maps and institutional affiliations.

(c) (i) Open Access This article is licensed under a Creative Commons Attribution 4.0 International License, which permits use, sharing, adaptation, distribution and reproduction in any medium or format, as long as you give appropriate credit to the original author(s) and the source, provide a link to the Creative Commons license, and indicate if changes were made. The images or other third party material in this article are included in the article's Creative Commons license, unless indicated otherwise in a credit line to the material. If material is not included in the article's Creative Commons license and your intended use is not permitted by statutory regulation or exceeds the permitted use, you will need to obtain permission directly from the copyright holder. To view a copy of this license, visit http://creativecommons.org/ licenses/by/4.0/.

(C) The Author(s) 2017 